\title{
Interbank Market Friction-Induced Holdings of Precautionary Liquidity - Implications for Bank Loan Supply and Monetary Policy Implementation
}

\author{
Monika Bucher · Achim Hauck · Ulrike \\ Neyer
}

Received: date / Accepted: date

\begin{abstract}
We analyse the impact of overnight interbank market frictions on bank loan supply when banks face idiosyncratic liquidity risk and discuss resulting implications for monetary policy implementation. Sufficiently pronounced interbank market frictions imply that banks hold positive or negative precautionary liquidity. Holding positive (negative) precautionary liquidity means that banks hold more (less) liquidity than they expect to need. As holding precautionary liquidity is costly, interbank market frictions negatively influence bank loan supply. However, by means of its standing facilities, the central bank not only offers an alternative to using the interbank market but also determines the costs of friction-induced holdings of positive or negative precautionary liquidity. Therefore, the facilities allow the central bank to influence banks' expected liquidity costs, and thereby their loan supply, so that interbank market frictions need not be an impediment to monetary policy transmission.
\end{abstract}

Keywords interbank market · monetary policy · precautionary liquidity · monetary policy implementation · interest corridor · loan supply

JEL Codes E52 E58 G21

Monika Bucher

Deutsche Bundesbank, Wilhelm-Epstein-Str. 14, 60431 Frankfurt am Main, Germany. (The views expressed in this paper represent the authors' personal opinions and do not necessarily reflect the views of Deutsche Bundesbank or the Eurosystem.)

Achim Hauck

Nottingham Trent University, Department of Economics, Nottingham Business School, 50 Shakespeare Street, Nottingham, United Kingdom NG1 4FQ, UK.

Ulrike Neyer

Heinrich Heine University Düsseldorf, Department of Economics, Universitätsstraße 1, 40225 Düsseldorf, Germany

Tel.: +49-211-8111511

E-mail: ulrike.neyer@hhu.de 


\section{Introduction}

Until the outbreak of the financial crisis in 2008, the Eurosystem ${ }^{1}$ used to provide just enough liquidity to euro area credit institutions sufficient to cover the structural liquidity deficit of the banking sector as a whole. ${ }^{2}$ Different bank-individual liquidity needs were balanced efficiently through the interbank market for reserves at an interbank rate fluctuating close to the rate on the Eurosystem's main refinancing operations, i.e. to the rate on those credit operations by which the Eurosystem provided the bulk of liquidity to the banking sector. The overnight interbank market thus played an important role for monetary policy implementation. It served as the starting point of the monetary policy transmission mechanism. By steering the interest rate in this market, the central bank aimed to affect banks' funding costs in order to influence bank loan supply and thereby aggregate demand and thus the price level in the euro area.

However, with the beginning of the financial crisis, the seizing up of the overnight interbank market could be observed. ${ }^{3}$ For various reasons, trading in this market became more expensive. Search costs increased as finding counterparties with matching liquidity needs became more difficult due, for example, to reduced credit lines among banks. ${ }^{4}$ Also, asymmetric information costs rose as uncertainty about the credit risk of interbank lending increased so that banks intensified their costly creditworthiness checks of potential borrowers, who in turn increased the costly signaling of their creditworthiness. ${ }^{5}$ Another potential source of higher interbank trading costs may be found in new financial regulations. ${ }^{6}$ As the interbank thus was no longer able to distribute

\footnotetext{
${ }^{1}$ The term "Eurosystem" stands for the institution which is responsible for monetary policy in the euro area, namely the ECB and the national central banks in the euro area. For the sake of simplicity, the terms "ECB" and "Eurosystem" are used interchangeably throughout this paper.

${ }^{2}$ Euro area credit institutions need central bank liquidity (reserves) for three reasons: to fulfill the reserve requirements imposed by the Eurosystem, to cope with so called autonomous factors (such as banknotes in circulation), and to settle payment transactions in the form of deposit transfers between bank customers of different institutions. The first two factors create a structural liquidity deficit of the banking sector which can only be covered by the Eurosystem. The deposit transfer payments, on the other hand, do not contribute to this liquidity deficit as resulting different bank-individual liquidity needs can be balanced through an interbank market. For a description of the credit institutions' demand for and the Eurosystem's supply of liquidity before the financial crisis see European Central Bank (2002).

${ }^{3}$ For a recent documentation on stress in the overnight interbank market in the euro area over the course of the financial and sovereign debt crisis in Europe see, for example, Frutos et al (2016).

${ }^{4}$ There are a couple of recently published papers that have developed interbank market models dealing with the search for suitable counterparties and bilateral negotiations. See, for example, Afonso and Lagos (2015), Bech and Monnet (2016), and Vollmer and Wiese (2016).

${ }^{5}$ Consequences of asymmetric information in interbank markets are analyzed, for example, in Freixas and Jorge (2008) and Heider et al (2015).

${ }^{6}$ For a recent discussion on the influence of new financial regulations on interbank market conditions see, for example, Bech and Keister (2013), Jackson and Noss (2015), Bank for
} 
liquidity efficiently, banks increased their demand for reserves and started to accumulate large amounts of liquidity on their accounts with the central bank. This was possible as with the beginning of the financial crisis, the Eurosystem gave banks access to unlimited central bank liquidity at a fixed rate against eligible collateral. The high levels of excess liquidity observed in the euro area during the period from 2008 until 2012 were thus mainly demand-driven (Baldo et al, 2017). Banks actively demanded relatively huge amounts of liquidity, and this demand was totally satisfied by the Eurosystem. ${ }^{7}$ This paper refers to such demand-driven excess liquidity holdings being the consequence of an imperfectly functioning interbank market for reserves.

Due to the importance of this interbank market for monetary policy implementation, the observed stress in this market raised concerns about the central bank's ability to actually influence bank loan supply and triggered a heated debate on the potential impairment of the transmission mechanism of monetary policy and the consequences for monetary policy implementation. ${ }^{8}$ Our paper aims to contribute to this debate by analysing the impact of overnight interbank market frictions on bank loan supply and by discussing resulting implications for monetary policy implementation. We develop a theoretical model in which banks have to decide on their loan supply to the non-banking sector in the presence of bank idiosyncratic liquidity risk and interbank market frictions. We capture the frictions in the form of broadly defined transaction costs. These costs may include search costs, asymmetric information costs and regulatory costs, i.e., similar to Bartolini et al (2001), we argue that frictions make interbank trading more expensive. ${ }^{9}$

International Settlements, Committee on the Global Financial System (2015), and Bindseil (2016).

${ }^{7}$ Since 2015, excess liquidity in the banking sector has increased again and has reached by now (October 2018) unprecedented levels. However, this rise in excess liquidity is largely supply-driven as it is mainly the result of the Eurosystem's large-scale asset purchases which started in 2015 to address the risks of a too prolonged period of low inflation (Baldo et al, 2017).

${ }^{8}$ The importance of the interbank market for the implementation and the transmission of monetary policy is pointed out, for example, by Heider et al (2015) and Frutos et al (2016) before they analyze the mal-functioning of this market in more detail. Freixas and Jorge (2008) show that an imperfect interbank market may lead to rationing in credit markets and thus may reinforce the impact of monetary policy on aggregate investment. Iyer et al (2014) argue that during the financial crisis, problems in the European interbank market had a negative impact on credit supply of those banks depending on interbank borrowing. And the ECB points out that a mal-functioning interbank market is a problem for the transmission of monetary policy and refers to interbank market problems when justifying the introduction of non-standard monetary policy measure (see, for example, European Central Bank (2009, 2010)).

${ }^{9}$ In our model, there is not an adverse selection problem due to asymmetric information as this would mean that lending to the overnight interbank market involves credit risk. However, in the overnight interbank market, lenders are typically unwilling to expose themselves to any counterparty credit risk as even for the slightest risk, the adequate interest rate is much higher than the rate on the alternative liquidity source, the central bank's lending facility (see Hauck and Neyer (2014) for a numerical example). We thus assume that asymmetric information leads to costly creditworthiness checks and costly signaling to 
We find that interbank market frictions negatively influence bank loan supply. However, they do not impede the transmission mechanism of monetary policy. By means of its standing facilities, the central bank is able to influence banks' expected liquidity costs, and thus their loan supply, also in the presence of significant, even prohibitive interbank market transaction costs. The reason is twofold. First, with its standing facilities the central bank provides an alternative to using the costly interbank market. ${ }^{10}$ Second, by setting the rates on its facilities the central bank determines the costs and therefore the amount of friction-induced holdings of positive or negative precautionary liquidity. The more precautionary liquidity (in absolute terms) banks hold, the lower their expected liquidity costs are. In our theoretical analysis, banks' holdings of precautionary liquidity play a crucial role. Banks will hold precautionary liquidity if interbank market frictions are sufficiently strong and if the expected costs of a liquidity deficit and of a liquidity surplus are asymmetric. Frictions will be sufficiently strong if they make interbank trading so expensive that at least one market side prefers to use the central bank's facilities, unless the other market side is willing to bear a higher share of the transaction costs. The asymmetric expected costs of a deficit and of a surplus may result from different probabilities of facing a deficit/surplus, stigma or regulatory costs which are relevant only to one interbank market side, or an asymmetric interest corridor formed by the rates on the central bank's standing facilities around the main policy rate. If banks expect a deficit to be more expensive than a surplus, they will hold positive precautionary liquidity, i.e., more liquidity than they expect to need. Otherwise they will hold negative precautionary liquidity.

Our model captures main elements of the ECB's monetary policy toolkit: its main refinancing operations and its two standing facilities. The main refinancing operations are credit operations with a maturity of one week by which the Eurosystem provides reserves to the banking sector. Starting from October 2008 the amount of this liquidity provision was not restricted, but, against adequate collateral, banks received the amount they asked for (fixed-rate full allotment). The two standing facilities, a deposit facility and a lending facility, allow banks to balance their overnight liquidity needs. The interest rates on the facilities form a corridor around the rate on the main refinancing operations with a deposit rate that is lower and a lending rate that is higher than the main refinancing rate. Before the Great Recession, the width of this corridor was 200 basis points. Starting from May 2009 the Eurosystem reduced the width first symmetrically and later asymmetrically with the main refinancing

eliminate the asymmetries, so that those overnight interbank market transactions actually taking place are risk-free.

${ }^{10}$ If banks make use of this alternative, the central bank will become an intermediary between liquidity deficit and surplus banks, i.e., interbank market activities will decline. In this context Bindseil (2016), among others, points to the trade-off between the central bank's aim to control the interbank rate (narrow interest corridor) and to maintain market activities (wide interest corridor). In the same vein, in our model a trade-off between boosting bank loan supply and maintaining interbank market activity could be discussed. While our paper does not broach this trade-off, it does focus on the impact of monetary policy measures on bank loan supply. 
rate moving closer to the deposit rate. As of October 2018, the width of the corridor amounts to 65 basis points. Since the interbank market froze during the financial crisis both the deposit and the lending facilities have been used to a considerable extent. Whereas the lending facility is now less frequently used, banks still place a significant share of their excess reserves in the deposit facility even though the deposit rate is negative. ${ }^{11}$

Based on our model, we can draw some conclusions for the ECB's monetary policy and bank loan supply in the euro area. From the beginning of the financial crisis up to now (October 2018) the ECB has not only significantly reduced banks' expected liquidity costs by lowering its key interest rates. By reducing the width of the corridor and switching to an asymmetric corridor later on, it has also lowered banks' friction-induced liquidity costs substantially. The ECB's monetary policy has thus prevented interbank market frictions from becoming an impediment to the transmission mechanism of monetary policy. Consequently, these frictions have not been responsible for weak bank lending observed in the euro area.

It should be noted that although our model captures main elements of the Eurosystem's operational framework, our main results also apply to other central banks' operational frameworks. Crucial is that banks facing stochastic deposit flows are induced to use the central bank's facilities to balance their actual liquidity position. ${ }^{12}$

The rest of this paper is organised as follows. Section 2 presents related literature. Section 3 describes the framework of the model. Section 4 derives the optimal liquidity management and optimal loan supply of an individual bank. Depending on the extent of interbank market frictions, three possible aggregate equilibria are presented in Section 5. Section 6 discusses the impact of different monetary policy measures on bank loan supply. Section 7 concludes the paper.

\section{Related Literature}

Our paper contributes to three strands of literature. The first strand analyzes how far frictions in financial markets influence the impact of monetary policy on bank lending. A huge part of this literature considers asymmetric information in credit markets and argues that these frictions amplify the effects of monetary policy on bank lending and therefore on aggregate demand. ${ }^{13}$

\footnotetext{
${ }^{11}$ For a detailed description of the Eurosystem's operational framework see, for example, European Central Bank (2012a) and the ECB's website. For a description of a use of the Eurosystem's instruments since 2009 see Eser et al (2012) and Alvarez et al (2017).

${ }^{12}$ In the USA, for example, banks can use the Federal Reserve System's discount window to balance a liquidity deficit, which is comparable to the Eurosystem's lending facility. Furthermore, the Federal Reserve System pays interest on excess reserves (IOER rate). This procedure is comparable to the remuneration of reserves in the Eurosystem's deposit facility.

${ }^{13}$ For a survey on this credit view of monetary policy see, for example, Boivin et al (2010) and Peek and Rosengreen (2012). One part of this credit view of monetary policy, the balance sheet channel, focuses on the impact of monetary policy on borrowers' net worth.
} 
The second strand of related literature deals with interbank market frictions. Until the outbreak of the financial crisis in 2007, the overnight interbank market was typically regarded as frictionless. As a result, papers dealing with this issue were rather rare. One of these papers is by Bartolini et al (2001). They consider interbank market transaction costs and show that these costs are responsible for a systematically high interbank rate at the end of reserve maintenance periods. However, the financial crisis inspired a rapidly growing literature dealing with interbank market imperfections. Freixas and Jorge (2008) show that asymmetric information about credit risk in the interbank market may induce the rationing of firms in credit markets, which leads first to a large impact of monetary policy on aggregate investment given the small interest elasticity of investment and second to a stronger impact on banks with less liquid balance sheets. Heider et al (2015) argue that asymmetric information about credit risk in the interbank market induces banks to hoard liquidity which may result in either adverse selection or a dry-up in the interbank market. However, banks may learn about counterparty credit risks by repeatedly trading with each other so that the asymmetric information problem may be mitigated. In an empirical analysis of the German unsecured overnight interbank market, Bräuning and Fecht (2017) determine the impact of such relationship lending on banks' ability to access liquidity. Iyer et al (2014) show that the contractionary effect of the impaired functioning of the Portuguese interbank market during the crisis on bank loan supply was stronger with banks which had less relationship lending. Besides Heider et al (2015), there are further papers that analyze how far interbank market frictions induce banks to hold precautionary liquidity. Ashcraft et al (2011) show that credit constraints and limited participation in combination with possible payment shocks can explain the precautionary holding of liquidity. Referring to the term interbank market, Acharya and Skeie (2011) trace precautionary liquidity holdings of potential lending banks back to the rollover risk of their own debt. Considering the overnight interbank market in the UK, Acharya and Merrouche (2012) argue that impaired markets for wholesale funding also lead to precautionary holding of liquidity. Allen et al (2009) show that if uncertainty about aggregate liquidity demand compared to idiosyncratic demand is sufficiently high, banks will start to hoard liquidity.

The third strand of related literature deals with the interbank market and monetary policy implementation. ${ }^{14}$ For monetary policy implementation the overnight interbank market plays a crucial role. The seminal paper in this literature is by Poole (1968). He develops a stochastic bank reserve management

Seminal papers dealing with the balance sheet channel are Bernanke and Gertler (1989) and Bernanke et al (1999). The other part of the credit view, the bank lending channel, focuses on the impact on bank deposits. Important papers dealing with this traditional bank lending channel are Gertler and Gilchrist (1993) and Kashyap and Stein (1995). Disyatat (2011) shows that a greater reliance on market-based funding instead of deposits creates a new approach to the bank lending channel.

${ }^{14}$ For a survey see, for example, Friedman and Kuttner (2011). By describing and discussing different parts of a central bank's operational framework, Bindseil (2014) gives a broad survey of monetary policy implementation in times of non-crisis and crisis. 
model in which banks are subject to a late payment shock, i.e., a liquidity shock which occurs after the closure of the interbank market. Banks ending the day with a liquidity deficit have to borrow from the central bank at a rate that is high relative to the interbank rate. Those banks ending with a liquidity surplus face forgone interest revenues from lending to the interbank market. Under uncertainty the banks, aiming to maximise their expected profit, thus have to decide on their optimal interbank market transactions. Poole's idea of using a late payment shock to introduce uncertainty into a bank reserve management model has been taken up by lots of papers. ${ }^{15}$ Generally, prior to the outbreak of the financial crisis in 2007, the literature on monetary policy implementation focuses on specific institutional aspects to explain interbank market participant behaviour and determinants of the interbank rate. Ho and Saunders (1985) and Clouse and Dow (2002), for example, consider major institutional characteristics of the US federal funds market. With respect to the federal funds market, a huge part of the literature analyzes why the federal funds rate fails to follow a martingale within the reserve maintenance period. ${ }^{16}$ A bulk of the literature on monetary policy implementation in the euro area deals with the under- and overbidding behaviour in the Eurosystem's main refinancing operations which could be observed in the first years of the European Monetary Union. ${ }^{17}$ Apart from this, there are papers that examine the consequences of alternative monetary policy implementation measures. Nautz (1998) shows that the central bank can influence the interbank market rate by being more or less vague about its future monetary policy. Välimäki (2001) analyzes the effects of alternative tender procedures with respect to the Eurosystem's refinancing operations. Neyer and Wiemers (2004) refer to the collateral framework. They show that differences in banks' opportunity costs of holding collateral form a rationale for the existence of an interbank market for reserves. Neyer (2009) demonstrates that a specific remuneration of required reserves increases the flexibility of monetary policy. Since 2008 a huge literature has evolved dealing with monetary policy implementation during the financial crisis. ${ }^{18}$ Borio and Disyatat (2009) emphasise the importance of the central bank balance sheet size in the implemented non-standard policy measures. Cheun et al (2009) consider changes to the collateral frameworks of the Eurosystem, the Federal Reserve System, and the Bank of England. Lenza et al (2010) describe the different ways in which these three central banks generally conducted monetary policy during the financial crisis. Eisenschmidt et al (2009)

\footnotetext{
${ }^{15}$ See, for example, Furfine (2000), Bindseil et al (2006), Whitesell (2006), and Bech and Monnet (2016).

${ }^{16}$ See, for example, Campbell (1987), Hamilton (1996), Clouse and Dow (1999), Furfine (2000), and Bartolini et al (2001, 2002).

${ }^{17}$ Under- and overbidding behaviour refers to a bidding behaviour in which total bids significantly deviate from the Eurosystem's benchmark allotment. Analyses with respect to this under- and overbidding behaviour can be found in, for example, Ayuso and Repullo (2001, 2003), Ewerhart (2002), Nautz and Oechssler (2003, 2006), and Bindseil (2005).

${ }^{18}$ For a description of the implementation of monetary policy by the Eurosystem in response to the financial crisis, see European Central Bank (2009, 2010, 2011, 2012b, 2014, 2015).
} 
analyze the relatively aggressive bidding behaviour of banks in the ECB's main refinancing operations at the beginning of the financial turmoil. Referring to the first part of the financial crisis (until 2008) too, Cassola and Huetl (2010) assess the effectiveness of monetary policy implementation during that time. Hauck and Neyer (2014) develop a theoretical model that considers the main institutional features of the ECB's operational framework, which has been in place since September 2008 to explain several stylized facts observed during the financial crisis. Bech and Monnet (2016) develop a search-based model of an over-the-counter (OTC) interbank market which also allows to explain several stylized facts observed in the euro area between 2009 and 2015. In their model, banks are exposed to settlement risk, which is comparable to Poole's late payment shock. This risk is responsible for the banks' use of the central bank's standing facilities. The literature emphasizing an active role of the central bank's standing facilities in monetary policy implementation is still rather new. Pérez-Quirós and Rodríguez-Mendizábal (2006) show that the standing facilities offered by the ECB, in combination with its minimum reserve system, is an effective instrument to stabilise the interbank rate. Whitesell (2006) looks at a minimum reserve system and standing facilities as two alternative regimes for controlling overnight interest rates. Berentsen and Monnet (2008) develop a general equilibrium framework and show that changing the rates on these facilities may be actively used as a monetary policy instrument. Colliard et al (2017) develop a core-periphery model of an OTC overnight interbank market. They argue that in a crisis a segmentation between core and periphery markets leads to a problematic dispersion of interbank rates, requiring the central bank to implement a floor operating system with respect to their interest corridor. ${ }^{19}$ Using a theoretical model, Link and Neyer (2017) show that interbank market transaction cost heterogeneity (bank- and time-specific) leads to interbank rate volatility, and they discuss possibilities for the central bank to control the volatility using alternative interest corridor systems. Alper et al (2013), Kara (2016), and Küçük et al (2016) describe and discuss the active role assigned to the interest corridor in the operational framework of the Turkish Central Bank that uses the interest corridor to smooth the volatility of cross-border capital flows, i.e., as a monetary policy tool for macroprudential purposes.

Our paper combines all three described strands of literature. The novelty of our paper is that it provides a theoretical model that explicitly links bank credit supply to interbank market frictions considering main elements of the Eurosystem's operational framework. This allows us to discuss implications for monetary policy implementation. With respect to the latter, we emphasise the active role of the central bank's standing facilities. We argue that they not only provide an alternative to the costly interbank market for balancing liquidity positions but the facility rates also determine the costs of frictioninduced holdings of positive or negative precautionary liquidity.

\footnotetext{
${ }^{19}$ In a floor operating system the central bank injects ample liquidity into the banking system, so that excess liquidity drives the interbank rate down to its lower bound, the rate on the deposit facility.
} 


\section{Setup}

The aim of the model is to shed light on lending and reserve management decisions of commercial banks in a setting that is institutionally rich in terms of a central bank's monetary policy tools and interbank market frictions. The model is short term in nature. It captures the duration of a central bank's refinancing operation. Our model economy is populated by three types of agents. There is a central bank, a continuum of measure one of price-taking, riskneutral commercial banks, and a large number of bank customers.

\subsection{Bank Loan Supply, Banks' Money Creation, and Banks' Liquidity Needs}

The commercial banks offer loans to their customers. The loan supply of an individual commercial bank is denoted by $L_{b}$. The interest rate a customer has to pay for a bank loan is $i^{L}$. Managing these loans generates costs $\frac{1}{2} \lambda L_{b}^{2}$ for a bank. The quadratic form of this cost function captures the idea that loans differ in their complexity so that a bank adds the least complex loans to its portfolio first. By making loans, banks create money. They credit the respective amount to their customers' deposit accounts. Bank customers can hold this newly created money on their accounts or use it to make payments. They can pay in cash, in which case they have to withdraw the respective amount of cash from their accounts, or by bank transfer.

The share of the new money used for cash payments is captured by the currency ratio $c$. Consequently, each bank faces a loss of deposits due to cash withdrawals equal to $c L_{b}$. The cash withdrawals generate a structural liquidity deficit for the whole banking sector that can only be covered by the central bank, that is the monopoly producer of currency.

In contrast, bank transfer payments do not generate a structural liquidity deficit of the banking sector as a whole, as one bank's deposit outflow is another bank's deposit inflow. However, a single bank may lose or gain deposits. The loss or gain in deposits is expressed as share $\chi t_{b}$ of the remaining deposits $(1-c) L_{b}$ with $\chi$ being a scale parameter and $t_{b}$ denoting the bank transfer ratio. If $t_{b}>0$, the bank will face a deposit outflow due to the bank customers' transfer payments. If $t_{b}<0$, there will be a deposit inflow. To sum up, a bank's liquidity needs resulting from cash withdrawals and bank transfer payments are

$$
\left(c+(1-c) \chi t_{b}\right) L_{b}
$$

While the currency ratio is the same for each bank, the bank transfer ratio differs across banks. It is the realization of the random variable $\tilde{t}_{b}$ which is distributed across banks according to the distribution function $G\left(t_{b}\right)$, with density $g\left(t_{b}\right)$, support $\left[t^{\min }, t^{\max }\right]$, and $-1 \leq t^{\min }<0<t^{\max } \leq 1$. The scale parameter $\chi$ with $\chi \in\left(0, \frac{1}{t^{\max }}\right]$ reflects the dispersion of the distribution of $\chi t_{b} \cdot{ }^{20}$ As a deposit outflow of one bank corresponds to a respective inflow of

\footnotetext{
${ }^{20}$ As the share $\chi t_{b}$ cannot exceed one, $\chi$ is restricted to $\frac{1}{t^{\max }}$.
} 
another bank, the expected value of a bank's transfer ratio is

$$
E\left[t_{b}\right]=\int_{t^{\min }}^{t_{\max }} t_{b} g\left(t_{b}\right) d t_{b}=0 .
$$

In our analysis we have to specify whether $G(0)$ is higher, smaller or equal to 0.5 . Further specifications of $G\left(t_{b}\right)$ are not necessary. If $G(0)=0.5$, the probability that a bank faces a deposit outflow will equal the probability of facing a deposit inflow. If $G(0)<0.5(G(0)>0.5)$, the probability of facing a deposit outflow is larger (smaller) than the probability of facing a deposit inflow. Figure 1 illustrates three possible distributions of $\tilde{t}_{b}$ implying different probabilities of facing a deposit outflow and a deposit inflow respectively. If there is a symmetric distribution of $\tilde{t}_{b}$ around $t_{b}=0$, the probability of facing a deposit outflow will equal the probability of facing a deposit inflow, $G(0)=0.5$. If there is a right-skewed (left-skewed) distribution of $\tilde{t}_{b}$, the probability of facing a deposit inflow is larger (smaller) than of facing a deposit outflow, $G(0)>0.5(G(0)<0.5)$. Note that Figure 1 only illustrates possible distributions of $\tilde{t}_{b}$. It is neither necessary to have a symmetric distribution of $\tilde{t}_{b}$ around $t_{b}=0$ to have $G(0)=0.5$, nor is it necessary to have a right-skewed (left-skewed) distribution to have $G(0)>0.5(G(0)<0.5)$. However, for the sake of a clearer presentation we will refer to a symmetric distribution of $\tilde{t}_{b}$ if $G(0)=0.5$ and to an asymmetric distribution if $G(0) \neq 0.5$. In our analysis, the possible asymmetry of the distribution plays a crucial role as it may imply that banks hold precautionary liquidity.

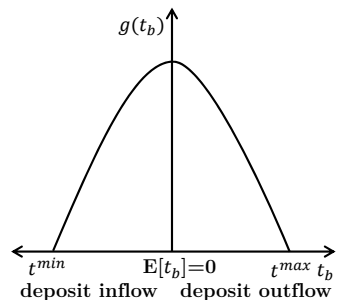

(a) Symmetric Distribution $G(0)=0.5$

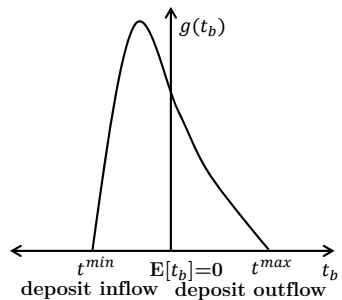

(b) Right-Skewed Distribution, $G(0)>0.5$

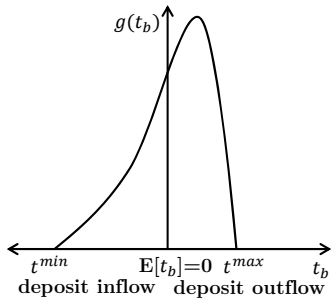

(c) Left-Skewed Distribution, $G(0)<0.5$

Fig. 1 Symmetric and Asymmetric Distributions of $\tilde{t}_{b}$

A bank balances its liquidity needs as specified in (1) by transacting with the central bank and/or in the interbank market. To obtain liquidity from the central bank, a bank can participate in the central bank's refinancing operations and borrow the amount $R O_{b}$ at the policy rate $i^{R O}$. The central bank fully satisfies a bank's liquidity demand in these operations. A bank can also use a lending facility to borrow $L F_{b}$ at the rate $i^{L F} \cdot{ }^{21}$ In addition, it can

\footnotetext{
${ }^{21}$ Generally, credit operations with the central bank require adequate collateral. However, the collateralization of central bank credits would not qualitatively change our results (see section 6.5 for details) so that, for the sake of simplicity, we ignore this aspect in our model.
} 
place an amount $D F_{b}$ of liquidity in a deposit facility offered by the central bank at the rate $i^{D F}$. The rates on the facilities form a corridor around the policy rate $i^{R O}$ with $i^{L F}>i^{R O}>i^{D F}$.

A bank's position in the interbank market is $B_{b}$. If $B_{b}>0$, the bank will borrow the amount $B_{b}$ at the rate $i^{I B M}$. Conversely, $B_{b}<0$ indicates that the bank will lend the amount $\left|B_{b}\right|$ at this rate. If it borrows or lends in the interbank market, transaction costs $\gamma\left|B_{b}\right|$ will accrue, with $\gamma \geq 0$. These transaction costs are broadly defined as explicated in the introduction.

\subsection{Informational Structure, Sequence of Events, and Optimization Problem}

As our focus is on the loan supply and the liquidity management of commercial banks, we do not explicitly model how the interest rate $i^{L}$ for bank loans is determined or the central bank sets its policy rates $i^{R O}, i^{L F}$ and $i^{D F}$. Instead, these rates are exogenously given and known to commercial banks. The same holds true with regard to the currency ratio $c$. The interbank rate $i^{I B M}$ is endogenously determined by supply and demand in this market. Our model is a one-period model with four events (see Figure 2). The timing and sequence of events during this period are as follows. At the beginning of this period, each bank decides on its loan supply $L_{b}$ and its borrowing from the central bank's refinancing operations $R O_{b}$. At this time, each bank does not yet know the realization of the transfer ratio and, therefore, its individual liquidity needs. However, it knows the distribution of $\tilde{t}_{b}$, so that it can form respective expectations. After each bank has decided on $L_{b}$ and $R O_{b}$, bank customers withdraw cash and make their bank transfer payments, i.e. each bank learns its individual $t_{b}$. Then, each bank has to decide whether to balance its resulting liquidity position by using the interbank market $B_{b}$ and/or the central bank's facilities $L F_{b}$ and $D F_{b}$. At the end of the period, all contracts mature without default.

Our one-period model thus captures the euro area banks' liquidity management within the Eurosystem's operational framework over one week. The first event reflects the euro area banks' possibility to borrow liquidity from the Eurosystem's main refinancing operations at the beginning of a week. At each day during the rest of the week euro area banks face deposit inflows and outflows due to their customers' payments. Banks balance the resulting liquidity deficit/surplus overnight by using the interbank market and/or the central bank's facilities at the end of each day. Our model subsumes the daily deposit inflows/outflows and the interbank market/facility transactions during the rest of the week in two events: first, customers' payments and second, banks' use of the interbank market/central bank's facilities.

When deciding on its activities, each bank seeks to maximize its expected profit. ${ }^{22}$ The sequence of events implies that each bank faces a two-stage op-

\footnotetext{
${ }^{22}$ In our model setup, maximizing a bank's expected profit is equivalent to maximizing its expected final net wealth. At the beginning of the considered period, each bank has an empty balance sheet, i.e., its net wealth is zero. During the period, a bank grants loans,
} 


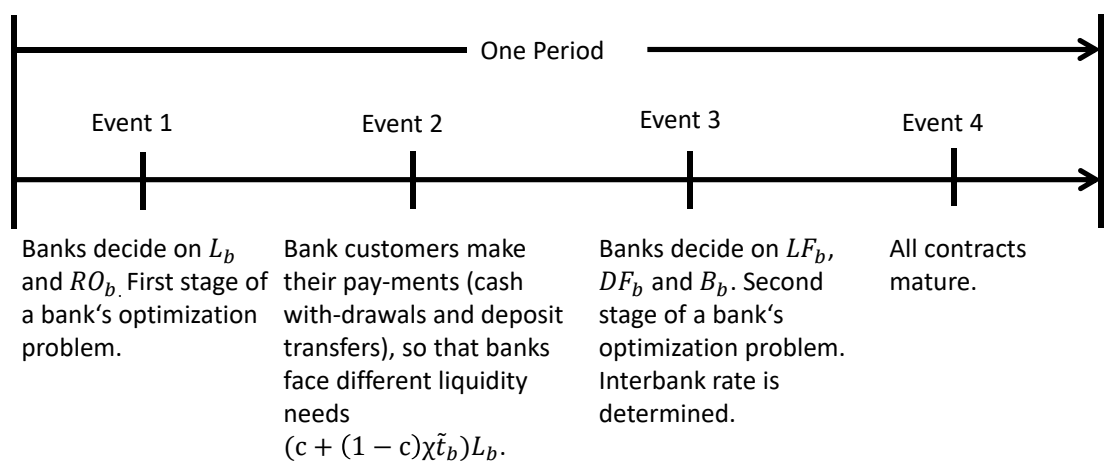

Fig. 2 Sequence of Events within a Period

timization problem that can be solved by backward induction. At the second stage, a bank aims to minimise its liquidity costs $C_{b}$ of using the interbank market and the facilities, taking the interbank rate, the policy rates as well as its liquidity needs as given. This optimization problem reads

$$
\begin{aligned}
\min _{B_{b} \in \mathbb{R} ; L F_{b}, D F_{b} \in \mathbb{R}_{0}^{+}} C_{b}\left(B_{b}, L F_{b}, D F_{b}\right)= & i^{I B M} B_{b}+\gamma\left|B_{b}\right| \\
& +i^{L F} L F_{b}-i^{D F} D F_{b} \\
\text { s.t. } \quad\left(c+(1-c) \chi t_{b}\right) L_{b}-R O_{b}= & B_{b}+L F_{b}-D F_{b} .
\end{aligned}
$$

The first two components of the cost function (3) reflect a bank's costs and possible revenues resulting from its transactions in the interbank market. The last two components represent its costs and revenues of using the central bank's facilities. Equation (4) describes a bank's balance sheet constraint. The left-hand side (LHS) reflects a bank's liquidity position at the beginning of the second stage, while the right-hand side (RHS) shows how this position is balanced.

At the first stage, each bank aims to maximise its expected profit. Denoting the respective objective function by $f$ and indicating the optimum variables of the second stage by the superscript opt, the optimization problem reads

$$
\begin{aligned}
\max _{L_{b}, R O_{b} \in \mathbb{R}_{0}^{+}} f\left(L_{b}, R O_{b}\right)= & i^{L} L_{b}-\frac{1}{2} \lambda L_{b}^{2}-i^{R O} R O_{b} \\
& -E\left[C_{b}\left(B_{b}^{o p t}, L F_{b}^{o p t}, D F_{b}^{o p t}\right)\right] .
\end{aligned}
$$

The first term of the objective function shows a bank's interest revenues from making loans to the non-banking sector. The second term describes its management costs. The third term reflects the liquidity costs which accrue from

creates deposits, conducts transactions with the central bank and with other banks on the interbank market. All these contracts mature at the end of the period. As there are no defaults, the bank's net wealth at the end of the period equals the profit it has made with these activities. 
borrowing from the central bank's refinancing operation. The last term represents the expected liquidity costs of using the interbank market and the central bank's facilities. Note that at this stage, bank customers have not made their payments yet. Accordingly, all banks are identical; they face the same optimization problem and thus form the same expectations about their subsequent liquidity needs.

To identify the subgame perfect equilibrium of the model, we proceed in two major steps. In a first step, we focus on the optimal behavior of a single bank, i.e. we solve its two-stage optimization problem, taking the interbank rate as given. ${ }^{23}$ Then, at the aggregate level, we determine the equilibrium interbank rate $i^{I B M}$ as well as aggregate borrowing from the refinancing operation and bank loan supply. We focus on equilibria in which the equilibrium interbank rate has two key characteristics. From an ex-post perspective, it balances aggregate supply and aggregate demand in the interbank market. From an ex-ante perspective, it is consistent with the interbank rate commercial banks base their decisions on at the first stage.

\section{Optimal Behaviour of a Single Bank}

To derive a bank's optimal behaviour, we solve the optimization problem by backward induction. First, we investigate the second stage determining a bank's optimal behaviour on the interbank market and its optimal use of the central bank's facilities. Then, we analyze the first stage and derive a bank's optimal borrowing from the central bank's refinancing operations and its optimal loan supply to the non-banking sector.

\subsection{Optimal Behaviour at the Second Stage}

Considering (1) and a bank's borrowing from the central bank's refinancing operations, its liquidity position at the beginning of the second stage is

$$
N_{b}:=\left(c+(1-c) \chi t_{b}\right) L_{b}-R O_{b} \gtreqless 0 .
$$

If $N_{b}>0$, the bank will face a liquidity deficit which has to be balanced by borrowing from the interbank market and/or the lending facility. Therefore, the bank compares the marginal costs of borrowing from the interbank market given by $i^{I B M}+\gamma$ with those of using the lending facility, which are $i^{L F}$. The bank will cover its total liquidity deficit by borrowing from the lending facility if $i^{I B M}+\gamma>i^{L F}$. If $i^{I B M}+\gamma \leq i^{L F}$, it will borrow exclusively from the interbank market. If $N_{b}<0$, there will be a liquidity surplus. The excess liquidity will be placed in the interbank market or the deposit facility

\footnotetext{
${ }^{23}$ Note that we can take the interbank rate as given because a commercial bank can conclude from its own first-stage behaviour what the aggregate liquidity position in the banking sector, and thus the consistent equilibrium interbank rate, will be at the second stage.
} 
depending on which alternative provides the largest marginal revenues. Note that if the marginal costs (marginal revenues) of using the interbank market and of using the central bank's lending (deposit) facility are identical, the bank will obviously be indifferent between both options. However, in such a case we break ties in favor of the interbank market. If the bank's liquidity position is balanced $\left(N_{b}=0\right)$, the bank will use neither the interbank market nor the facilities. From (6) we can infer that $N_{b}=0$ if

$$
t_{b}=\frac{R O_{b}-c L_{b}}{(1-c) \chi L_{b}}=: \bar{t}_{b} .
$$

We denote $\bar{t}_{b}$ as the critical bank transfer ratio. This critical ratio plays a crucial role in our analysis as it determines whether a bank faces a liquidity deficit or surplus at the beginning of the second stage.

\subsection{First Stage: Optimal Borrowing from the Refinancing Operations}

The equilibrium interbank rate $i^{I B M *}$ must be in the interval $\left[i^{R O}-\gamma, i^{R O}+\gamma\right]$ (see comment to proof of Lemma 1 in the appendix). For the sake of a clearer presentation, we thus exclude interbank rates $\notin\left[i^{R O}-\gamma, i^{R O}+\gamma\right]$ already at this stage. Solving the optimization problem given by (5) for $R O_{b}^{o p t}$, we then obtain

Lemma 1 Suppose that the non-negativity constraint on $\mathrm{RO}_{b}$ is not binding. ${ }^{24}$ Then, differentiating $f$ partially with respect to $R_{b}$ gives the first-order condition (FOC) for a bank's optimal borrowing from the refinancing operations $R O_{b}^{\text {opt }}$ :

$$
\begin{aligned}
& i^{R O}= \max \left\{i^{I B M}-\gamma, i^{D F}\right\} G\left(\bar{t}_{b}^{o p t}\right) \\
&+\min \left\{i^{I B M}+\gamma, i^{L F}\right\}\left(1-G\left(\bar{t}_{b}^{o p t}\right)\right) \\
& \text { with } \quad \bar{t}_{b}^{o p t}=\frac{R O_{b}^{o p t}-c L_{b}^{o p t}}{(1-c) \chi L_{b}^{o p t}} .
\end{aligned}
$$

Proof See appendix.

The LHS of the FOC for a bank's optimal borrowing from the central bank's refinancing operations shows marginal costs, the RHS expected marginal revenues. ${ }^{25}$ With probability $G\left(\bar{t}_{b}^{\text {opt }}\right)$, a bank will face a liquidity surplus at the

\footnotetext{
${ }^{24}$ For the sake of a clearer presentation we do not explicitly consider the non-negativity constraint on $R O_{b}$ in the presentation of our model, but we will comment on this aspect if relevant (see footnotes 31 and 38 to 44 ).

${ }^{25}$ Note that at the first stage, a bank only forms expectations about the individual liquidity needs it will face at the second stage. The interbank rate is known for certain: All banks face exactly the same optimization problem at the first stage, so $R O_{b}^{\text {opt }}$ is identical for all banks. As there is no aggregate uncertainty, each bank therefore knows the aggregate liquidity endowment of the banking sector and thus the interbank rate for any $R O_{b}^{o p t}$.
} 
beginning of the second stage. In this case, any additional borrowing from the refinancing operations will generate additional interest revenues (net of possible transaction costs) as the additional surplus will be lent to the interbank market or placed in the deposit facility. With probability $1-G\left(\bar{t}_{b}^{\text {opt }}\right)$, a bank will face a liquidity deficit. Borrowing more from the refinancing operation at the first stage will reduce the liquidity deficit at the second stage, so that in this case marginal revenues in the form of avoided illiquidity costs will accrue.

For the following analysis some definitions are useful. First, we denote the interbank rate that balances marginal costs and expected marginal revenues of borrowing from the refinancing operations for $R O_{b}=c L_{b}$ by $\tilde{i}^{I B M}$. As $R O_{b}^{o p t}=c L_{b}^{o p t}$ implies that $\bar{t}_{b}^{o p t}=0$, it follows from (8) that

$$
\tilde{i}^{I B M}=i^{R O}-\gamma(1-2 G(0)) \text {. }
$$

Second, it is useful to distinguish deviations from a bank's expected liquidity needs. Considering (1) and (2), a bank's expected liquidity needs are $c L_{b}$. If a bank borrows more (less) than $c L_{b}$ from the refinancing operations, so that $\bar{t}_{b}>0\left(\bar{t}_{b}<0\right)$, it will thus hold positive (negative) precautionary liquidity. This leads us to

Lemma $2 A$ bank will hold positive (negative) precautionary liquidity if it expects for $R O_{b}=c L_{b}$, i.e., for $\bar{t}_{b}=0$, that a deficit will be more (less) expensive at the margin than a surplus, i.e., if

$$
\begin{aligned}
& \left(i^{R O}-\max \left\{i^{I B M}-\gamma, i^{D F}\right\}\right) G(0) \\
& \gtrless\left(\min \left\{i^{I B M}+\gamma, i^{L F}\right\}-i^{R O}\right)(1-G(0)) .
\end{aligned}
$$

The RHS of (11) presents the expected marginal costs of a deficit, the LHS of a surplus. From (11) we can conclude that if a bank holds precautionary liquidity, some kind of asymmetry will be involved. In our model, there are two possible sources of asymmetry: an asymmetric distribution of $\tilde{t}_{b}$ around zero $(G(0) \neq$ $0.5)$ and an asymmetric interest corridor $\left(i^{L F}-i^{R O} \neq i^{R O}-i^{D F}\right)$. However, one can think of other possible sources, such as stigma costs when borrowing from the interbank market or different transaction costs for interbank market lending and borrowing. Note that for the sake of a less complex presentation of the proofs of our propositions and results, we assume either the interest corridor or the distribution of $\tilde{t}_{b}$ to be asymmetric or both to be symmetric. ${ }^{26}$ Allowing for both to be asymmetric would not change our results. Rearranging (8), we obtain the FOC for optimal precautionary liquidity holdings:

$$
\begin{aligned}
& \left(i^{R O}-\max \left\{i^{I B M}-\gamma, i^{D F}\right\}\right) G\left(\bar{t}_{b}^{o p t}\right) \\
& =\left(\min \left\{i^{I B M}+\gamma, i^{L F}\right\}-i^{R O}\right)\left(1-G\left(\bar{t}_{b}^{o p t}\right)\right) .
\end{aligned}
$$

If a bank holds positive precautionary liquidity, the LHS of (12) will represent its expected marginal costs of holding this liquidity in the form of additional

\footnotetext{
${ }^{26} \mathrm{See}$, for example, the proof of Proposition 3 in the appendix.
} 
interest costs, while the RHS will show its expected marginal revenues in the form of avoided illiquidity costs. If a bank holds negative precautionary liquidity, the LHS of (12) will represent the expected marginal revenues of holding this liquidity in the form of avoided interest costs while the RHS will show respective expected marginal costs in the form of additional illiquidity costs.

\subsection{First Stage: Optimal Bank Loan Supply}

Solving the optimization problem given by (5) for $L_{b}^{o p t}$, we obtain

Lemma 3 Differentiating $f$ partially with respect to $L_{b}$ and using the result for optimal borrowing from the refinancing operations for a not binding nonnegativity constraint on $\mathrm{RO}_{b}$ given by (8), the FOC for a bank's optimal loan supply to the non-banking sector becomes

$$
\begin{aligned}
& \begin{array}{l}
i^{L}= \\
\quad \lambda L_{b}^{o p t}+c i^{R O} \\
\quad+\phi\left(\bar{t}_{b}^{o p t}\right)\left[\min \left\{i^{I B M}+\gamma, i^{L F}\right\}-\max \left\{i^{I B M}-\gamma, i^{D F}\right\}\right]
\end{array} \\
& \text { with } \bar{t}_{b}^{\text {opt }} \text { defined by (9) and } \\
& \phi\left(\bar{t}_{b}^{o p t}\right):=(1-c) \chi \int_{\bar{t}_{b}^{\text {opt }}}^{t^{\text {max }}} t_{b} g\left(t_{b}\right) d t_{b} .
\end{aligned}
$$

Proof See appendix.

The LHS of the FOC for a bank's optimal loan supply presents the marginal revenues of granting loans, the RHS expected marginal costs. The latter consist of the marginal management costs $\lambda L_{b}^{\text {opt }}$ and expected marginal liquidity costs. In the following, we will comment on the latter in more detail.

If a bank increases its loans by one unit, it will face additional certain liquidity needs $c$ because of additional cash withdrawals. These certain liquidity needs imply certain marginal liquidity costs $c i^{R O}$. Furthermore, the increase in loans implies additional uncertain liquidity needs due to uncertain bank transfer payments $(1-c) \chi \tilde{t}_{b}$. And although the respective expected additional liquidity needs are zero as $E\left[t_{b}\right]=0$, the bank's expected additional (net) liquidity costs will be positive if the possible deficit costs are higher than the possible surplus revenues (in this case, the term in square brackets in (13) will be positive).

We refer to these costs as the uncertain liquidity costs of granting loans. Note that if interbank trade is not impaired $(\gamma=0)$, uncertain liquidity costs of granting loans will be zero. The importance of these costs for a bank's optimal bank loan supply is reflected by the quantity and probability weight 
$\phi\left(\bar{t}_{b}^{o p t}\right) .{ }^{27}$ Consequently, the last term in the FOC (13) presents the expected marginal uncertain liquidity costs of granting loans.

In our analysis, it plays a crucial role that the weight $\phi\left(\bar{t}_{b}^{o p t}\right)$, and thus the expected marginal uncertain liquidity costs of granting loans, decrease in a bank's holdings of positive as well as negative precautionary liquidity:

$$
\frac{\partial \phi\left(\bar{t}_{b}^{o p t}\right)}{\partial \bar{t}_{b}^{o p t}}=-(1-c) \chi \bar{t}_{b}^{o p t} g\left(\bar{t}_{b}^{o p t}\right)\left\{\begin{array}{lll}
<0 & \text { if } \bar{t}_{b}^{o p t}>0 \\
>0 & \text { if } \bar{t}_{b}^{o p t}<0 .
\end{array}\right.
$$

\section{Aggregate Equilibrium}

\subsection{Interbank Rate}

After having clarified the optimal behaviour of an individual bank, we are now in a position to look at the aggregate equilibrium. ${ }^{28}$ At the second stage, each bank will only trade liquidity in the interbank market if this is more beneficial than using the respective facility. All variables and parameters determining this decision are the same for all banks. Consequently, if $i^{I B M}-\gamma<i^{D F}$, all banks with a liquidity surplus will place their excess liquidity in the deposit facility, while for $i^{I B M}+\gamma>i^{L F}$, all banks with a liquidity deficit will borrow the missing liquidity from the lending facility. There is thus an upper and a lower bound for the interbank rate given by

$$
\begin{aligned}
& \overline{i^{I B M}}=i^{L F}-\gamma, \\
& \underline{i^{I B M}}=i^{D F}+\gamma .
\end{aligned}
$$

These bounds for $i^{I B M}$ lead us to an upper threshold for $\gamma$. If $\gamma$ exceeds

$$
\overline{\bar{\gamma}}:=\frac{i^{L F}-i^{D F}}{2},
$$

the interbank market will break down. Transaction costs are prohibitively high. Both the upper and the lower bound for the interbank rate will become

${ }^{27}$ The weight of the deficit costs is $(1-c) \chi \int_{\bar{t}_{b}^{o p t}}^{t^{\max }} t_{b} g\left(t_{b}\right) d t_{b}$, the weight of the surplus revenues is $-(1-c) \chi \int_{t^{m i n}}^{\bar{t}_{b}^{o p t}} t_{b} g\left(t_{b}\right) d t_{b}$. Note that $\int_{\bar{t}_{b}^{o p t}}^{t^{m a x}} t_{b} g\left(t_{b}\right) d t_{b}=E\left[t_{b} \mid t_{b}>\bar{t}_{b}^{o p t}\right](1-$ $\left.G\left(t_{b}\right)\right)$ and that $-\int_{t_{\text {min }}}^{\bar{t}_{b}^{\text {opt }}} t_{b} g\left(t_{b}\right) d t_{b}=-E\left[t_{b} \mid t_{b}<\bar{t}_{b}^{\text {opt }}\right] G\left(t_{b}\right)$, so that the weights reflect the expected additional deficit/surplus and the respective occurrence probability. As $E\left[t_{b}\right]=0$, we have $-(1-c) \chi \int_{t^{\text {min }}}^{\bar{t}_{b}^{o p t}} t_{b} g\left(t_{b}\right) d t_{b}=(1-c) \chi \int_{\bar{t}_{b}^{o p t}}^{t^{\text {max }}} t_{b} g\left(t_{b}\right) d t_{b}$, which means that the weight with which possible deficit costs and surplus revenues enter the FOC is the same. Capturing this identical weight by $\phi\left(\bar{t}_{b}^{o p t}\right)$, the expected marginal uncertainty costs of granting loans simplify to the last term in (13).

${ }^{28}$ Note that we will derive the interbank market equilibrium. However, we will not derive a credit market equilibrium. Our focus is on the supply side of the credit market. We analyze how far the costs and the availability of liquidity (and thus monetary policy in general, its implementation in particular and the functioning of the interbank market) have an impact on bank loan supply. 
binding. This implies that the set of possible equilibrium interbank rates $I$ is given by

$$
I= \begin{cases}{\left[\underline{i^{I B M}}, \overline{i^{I B M}}\right]} & \text { if } \gamma \leq \overline{\bar{\gamma}}, \\ \{\} & \text { if } \gamma>\overline{\bar{\gamma}} .\end{cases}
$$

Denoting aggregate borrowing from the refinancing operations by $R O$, aggregate loan supply to the non-banking sector by $L$, and (subgame perfect) equilibrium variables by an asterisk, these considerations lead us to

Proposition 1 (a) If $\gamma>\overline{\bar{\gamma}}$, there will not be an active interbank market, $B^{*}=0$.

(b) If $\gamma \leq \overline{\bar{\gamma}}$, there will be an active interbank market at the second stage, and the subgame perfect equilibrium interest rate $i^{I B M *}$ at the first stage will be

$$
i^{I B M *}= \begin{cases}\frac{i^{I B M}}{\tilde{i}^{I B M}} \in & \text { if } R O^{*}>c L^{*}, \\ \frac{\text { if } R O^{*}=c L^{*},}{i^{I B M}} & \text { if } R O^{*}<c L^{*},\end{cases}
$$

where $\tilde{i}^{I B M}$ is defined by (10).

Proof See appendix.

Using Figure 3, we illustrate in more detail the equilibrium interbank rate given in Proposition 1. An interbank rate below $\overline{i^{I B M}}$ implies that all banks facing a liquidity deficit want to borrow their missing liquidity from the interbank market. Consequently, if $i^{I B M}<\overline{i^{I B M}}$, there will be a perfectly inelastic, strictly positive demand for liquidity under all three possible liquidity conditions in the banking sector presented in Figure 3. If $i^{I B M}>\overline{i^{I B M}}$, all deficit banks will prefer to borrow their liquidity from the central bank's lending facility, i.e. under all three possible liquidity conditions in the banking sector, interbank demand for liquidity will equal zero. If $i^{I B M}=\overline{i^{I B M}}$, banks are indifferent whether to borrow from the interbank or the central bank's lending facility, i.e. interbank demand at this rate is perfectly elastic. Analogously, there will be a perfectly inelastic, strictly positive supply of liquidity if $i^{I B M}>i^{I B M}$, interbank supply will equal zero if $i^{I B M}<\underline{i^{I B M}}$, and interbank supply will be perfectly elastic if $i^{I B M}=\underline{i^{I B M}}$. Which equilibrium interbank rate will be realised depends on the banking sector's liquidity position.

An aggregate liquidity deficit will arise if the banking sector's structural liquidity needs $c L^{*}$ exceed the aggregate amount borrowed from the refinancing operations $R O^{*}$. In this case, which is presented in Panel (a) of Figure 3, for all $i^{I B M}<\overline{i^{I B M}}$, there is an excess demand for liquidity, whereas for all $i^{I B M}>\overline{i^{I B M}}$ there is an excess supply. The equilibrium interbank rate, achieved through a process of tâtonnement, ${ }^{29}$ thus equals $\overline{i^{I B M}}$. To put it differently, if there is an aggregate liquidity deficit, competition for scarce liquidity will bring the interbank rate to its upper bound $\overline{i^{I B M}}$. At this upper

${ }^{29}$ For a formal analysis of this process see, for example, Andreu Mas-Colell and Michael D. Whinston and Jerry R. Green (1995, chapter 17). 
bound, there is still a liquidity deficit in the banking sector so that the central bank's lending facility has to be used.

The case of an aggregate liquidity surplus in the banking sector is illustrated in Panel (b). Analogously to the deficit-case, competition for limited lending possibilities in the interbank market will bring the interbank rate to its lower bound $i^{I B M}$. At this lower bound, there is still a liquidity surplus in the banking sector, so that liquidity has to be placed in the central bank's deposit facility.

If there is neither an aggregate liquidity deficit nor surplus $\left(R O^{*}=c L^{*}\right)$, see Panel (c), any rate within the interval $\left[\underline{i^{I B M}}, \overline{i^{I B M}}\right]$ can be consistent with an equilibrium at the second stage of the model. However, at the first stage, only $\tilde{i}^{I B M}$, as given by (10), will be the subgame perfect equilibrium interest rate for $R O^{*}=c L^{*}$. For all other $i^{I B M} \in I$, the expected marginal revenues of borrowing from the refinancing operations strictly deviate from marginal costs, inducing banks to borrow more or less than $c L$ from the refinancing operations, i.e. $\tilde{i}^{I B M} \in I$ will be the only subgame perfect equilibrium rate if liquidity conditions are balanced $\left(R O^{*}=c L^{*}\right)$. As there is neither a liquidity surplus nor deficit in the banking sector, none of the central bank's facilities is used.

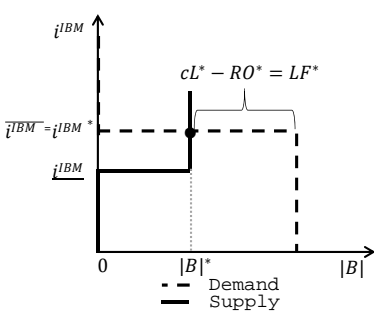

(a) Liquidity Deficit, $R O^{*}<c L^{*}$

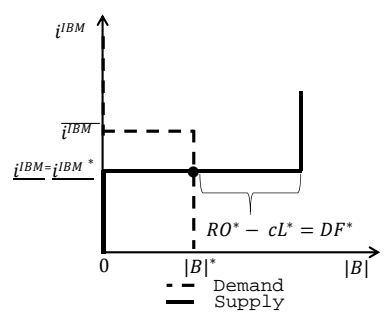

(b) Liquidity Surplus, $R O^{*}>c L^{*}$

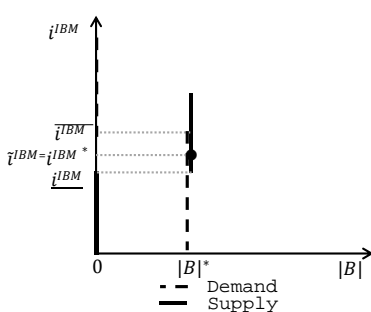

(c) Balanced Liquidity Conditions, $R O^{*}=c L^{*}$

Fig. 3 Interbank Market Equilibrium

Proposition 1 leads us to a further threshold for $\gamma:{ }^{30}$

$$
\bar{\gamma}:=\min \left\{\bar{\gamma}_{l}:=\frac{i^{R O}-i^{D F}}{2(1-G(0))}, \bar{\gamma}_{u}:=\frac{i^{L F}-i^{R O}}{2 G(0)}\right\} \leq \overline{\bar{\gamma}} .
$$

If $\gamma \leq \bar{\gamma}$, we will have $\tilde{i}^{I B M} \in I$, i.e., borrowing $R O=c L$ will balance the banks' expected marginal revenues and the marginal costs of borrowing from the refinancing operations. If $\bar{\gamma}<\gamma \leq \overline{\bar{\gamma}}$, this will no longer be the case, $\tilde{i}^{I B M} \notin I$. If $\bar{\gamma}=\bar{\gamma}_{l}<\gamma \leq \overline{\bar{\gamma}}$, we will have $\tilde{i}^{I B M}<\underline{i^{I B M}}$, and if $\bar{\gamma}=\bar{\gamma}_{u}<\gamma \leq$ $\overline{\bar{\gamma}}$, we will have $\tilde{i}^{I B M}>\overline{\overline{i^{I B M}}}$. This means that if $\overline{\bar{\gamma}<\gamma} \leq \overline{\bar{\gamma}}$, there will be an active interbank market, but for all $i^{I B M} \in I$ expected marginal revenues of

\footnotetext{
${ }^{30}$ One obtains $\bar{\gamma}_{l}$ by setting in (10) $\tilde{i}^{I B M}=\underline{i^{I B M}}$ and solving that equation for $\gamma$. Analogously, one obtains $\bar{\gamma}_{u}$ by setting in (10) $\tilde{i}^{I B} \bar{M}=\bar{i}^{I B M}$.
} 
borrowing from the refinancing operations will deviate from marginal costs for $R O=c L$. Banks will thus borrow more or less than $c L$ from the refinancing operations as we will show in the next section.

\subsection{Borrowing from the Refinancing Operations and Bank Loan Supply}

At the first stage, all banks face the same optimization problem, so that $R O_{b}^{o p t}$, $L_{b}^{o p t}$ and, therefore, also $\bar{t}_{b}^{o p t}$ are identical for all banks. As we have a continuum of banks of unit mass, these bank-individual optimal values correspond to the respective aggregate equilibrium variables $L^{*}$ and $R O^{*}$. Furthermore, we can use $\bar{t}^{\text {opt }}=\bar{t}^{*}$ as the critical equilibrium bank transfer ratio is identical for all banks. Depending on the level of interbank market transaction costs, three different equilibria indexed by $j=I, I I, I I I$ are possible. The main characteristics of these equilibria are:

- Equilibrium I: Low transaction costs, active interbank market, no precautionary liquidity holdings.

- Equilibrium II: High transaction costs, active interbank market, precautionary liquidity holdings.

- Equilibrium III: Prohibitively high transaction costs, inactive interbank market, possible precautionary liquidity holdings.

In the following, we will have a closer look at each of them.

\subsubsection{Equilibrium I: Low Transaction Costs}

Proposition 2 If $\gamma \leq \bar{\gamma}$ banks will exclusively use the interbank market to balance their liquidity position at the second stage, i.e.,

$R O^{* I}=c L^{* I}, \quad$ so that $\quad \bar{t}^{* I}=\frac{R O^{* I}-c L^{* I}}{(1-c) \chi L^{* I}}=0 \quad$ and $\quad i^{I B M * I}=\tilde{i}^{I B M} \in I$.

Proof See appendix.

If interbank market transaction costs are sufficiently low $(\gamma \leq \bar{\gamma})$, borrowing $R O=c L$ will balance banks' expected marginal revenues and the marginal costs of borrowing from the refinancing operations. Borrowing more (less) than $c L$ from the refinancing operations would result in an aggregate liquidity surplus (deficit) at the beginning of the second stage bringing the interbank rate to its lower (upper) bound. This low (high) interbank rate would imply that the expected marginal revenues of borrowing from the refinancing operations would strictly deviate from marginal costs. Consequently, if $\gamma \leq \bar{\gamma}$, $R O^{* I}=c L^{* I}$ will be the only feasible equilibrium.

Note that for this result a possible asymmetry of the interest corridor or of the distribution of $\tilde{t}_{b}$ does not play a role. The rates on the facilities are irrelevant for the banks' borrowing decision, as with $\gamma \leq \bar{\gamma}$ transaction costs are so low that no bank expects to use the central bank's facilities. With respect to a possible asymmetric distribution of $\tilde{t}_{b}$ consider that rising transaction 
costs increase the costs of both, balancing a surplus and a deficit. However, an asymmetric distribution of $\tilde{t}_{b}(G(0) \neq 0.5)$ means that for $R O=c L$ banks expect a deficit to be more or less probable so that rising transaction costs will increase the expected costs of a deficit more or less than those of a surplus. This should induce banks to hold positive or negative precautionary liquidity. However, as long as $\gamma \leq \bar{\gamma}$, there will be a change in $i^{I B M * I}=\tilde{i}^{I B M}$ exactly compensating the effect of an increase in $\gamma$ on marginal surplus and deficit costs, i.e., the price mechanism in the interbank market functions, so that $R O^{* I}=c L^{* I}$ remains the equilibrium solution. Formally, this can be seen by having a closer look at Lemma 1 . In case of an active interbank market, (8) can be rewritten as

$$
\begin{aligned}
& \left(i^{R O}-i^{I B M * j}+\gamma\right) G\left(\bar{t}^{* j}\right) \\
& =\left(i^{I B M * j}+\gamma-i^{R O}\right)\left(1-G\left(\bar{t}^{* j}\right)\right), \quad \text { with } j=I, I I .
\end{aligned}
$$

The LHS of (22) shows the expected marginal costs of a surplus, the RHS of a deficit. In Equilibrium I, $i^{I B M * I}=\tilde{i}^{I B M}$ as given by $(10)$ and $\bar{t}^{* I}=0$. An increase in $\gamma$ does not change either side as it is exactly compensated by a respective decrease in $i^{I B M * I}$.

Lemma 3 reveals that interbank market transaction costs are a crucial determinant of banks' expected marginal uncertain liquidity costs of granting loans. For $\gamma \leq \bar{\gamma}$, we have $R O^{* I}=c L^{* I}$, i.e. $\bar{t}^{* I}=0$, and all banks prefer to use the interbank market instead of the facilities. Expected marginal uncertain liquidity costs of granting loans thus simplify to

$$
\phi(0) 2 \gamma, \quad \text { where } \quad \phi(0)=(1-c) \chi \int_{0}^{t^{\max }} t_{b} g\left(t_{b}\right) d t_{b}
$$

If interbank trading is not impaired $(\gamma=0)$, expected marginal uncertain liquidity costs will be zero. Expected deficit costs and expected surplus revenues will be the same, both will equal the interbank rate. However, positive transaction costs mean higher costs when borrowing from the interbank market and lower revenues when lending to this market, i.e., they put a wedge between the costs and revenues of trading in the interbank market, leading to positive expected marginal uncertain liquidity costs.

\subsubsection{Equilibrium II : High Transaction Costs}

Proposition 3 (a) If $\bar{\gamma}=\bar{\gamma}_{l}<\gamma \leq \overline{\bar{\gamma}}$, banks will hold positive precautionary liquidity and will use the interbank market and the deposit facility to balance their liquidity position at the second stage, i.e.,

$$
R O^{* I I}>c L^{* I I}, \text { so that } \bar{t}^{* I I}=\frac{R O^{* I I}-c L^{* I I}}{(1-c) \chi L^{* I I}}>0 \text { and } i^{I B M * I I}=\underline{i^{I B M}} \text {. }
$$


(b) If $\bar{\gamma}=\bar{\gamma}_{u}<\gamma \leq \overline{\bar{\gamma}}$, banks will hold negative precautionary liquidity and will use the interbank market and the lending facility to balance their liquidity position at the second stage, i.e., ${ }^{31}$

$$
R O^{* I I}<c L^{* I I} \text {, so that } \bar{t}^{* I I}=\frac{R O^{* I I}-c L^{* I I}}{(1-c) \chi L^{* I I}}<0 \text { and } i^{I B M * I I}=\overline{i^{I B M}} .
$$

If interbank market transaction costs are sufficiently high $(\gamma>\bar{\gamma})$, possible asymmetries of the interest corridor or of the distribution of $\tilde{t}_{b}$ will be relevant for banks' optimal borrowing from the refinancing operations. Due to the asymmetries, banks expect a deficit to be more or less expensive than a surplus for $R O=c L$, so that they hold precautionary liquidity.

If we have a symmetric distribution of $\tilde{t}_{b}(G(0)=0.5)$ but an asymmetric interest corridor in the form of $i^{L F}-i^{R O}>i^{R O}-i^{D F}$, an increase in $\gamma$ beyond $\bar{\gamma}=\bar{\gamma}_{l}$ will imply that at first only banks with a liquidity surplus will no longer be willing to use the interbank market but will prefer the central bank's deposit facility, whereas for banks with a liquidity deficit, it will be still more beneficial to use the interbank market. To maintain an active interbank market, deficit banks thus accept having to also bear the transaction costs of the surplus banks. ${ }^{32}$ Note that if $\gamma$ exceeds $\overline{\bar{\gamma}}$, transaction costs will be so high, that deficit banks will no longer be willing to bear double transaction costs, but will prefer to use the central bank's facilities. The interbank market will break down (Equilibrium III). However, as long as we have an active interbank market, due to the higher transaction cost burden, banks expect a deficit to be more expensive than a surplus inducing them to hold positive precautionary liquidity, $R O^{* I I}>c L^{* I I} .^{33}$ Analogously, banks will hold negative precautionary liquidity $\left(R O^{* I I}<c L^{* I I}\right)$ if $i^{L F}-i^{R O}<i^{R O}-i^{D F}$ and if $\bar{\gamma}=\bar{\gamma}_{u}<\gamma \leq \overline{\bar{\gamma}}$.

Let us turn next to the case of a symmetric interest corridor and an asymmetric distribution of $\tilde{t}_{b}$ in the form of $G(0)<0.5$. The asymmetric distribution means that for $R O=c L$ banks expect a deficit to be more probable than a surplus. As long as $\gamma \leq \bar{\gamma}=\bar{\gamma}_{l}$, the resulting stronger impact of an increase in $\gamma$ on expected marginal deficit costs rather than on surplus costs will be compensated by a respective decrease in $\tilde{i}^{I B M}$ (Equilibrium I). However, if $\gamma$ reaches $\bar{\gamma}_{l}$, further decreases in $\tilde{i}^{I B M}$ will no longer be possible, the price mechanism in the interbank market will not function any longer as the lower bound for $i^{I B M}$ will become binding. Then, further increases in $\gamma$ mean that

\footnotetext{
${ }^{31}$ Note that if banks hold negative precautionary liquidity and if the non-negativity constraint on $R O_{b}$ binds, $R O^{* I I}=0$ and $\bar{t}^{* I I}=\underline{t}=-c /(1-c) \chi<0$ (see proof of Lemma 1 in the appendix).

${ }^{32}$ In this case, balancing a liquidity deficit in the interbank market costs $i^{I B M * I I}+\gamma=$ $\underline{i^{I B M}}+\gamma=i^{D F}+2 \gamma$.

${ }^{33}$ If $\bar{\gamma}=\bar{\gamma}_{l}<\gamma \leq \overline{\bar{\gamma}}, \tilde{i}^{I B M}<\underline{i^{I B M}}$ but $I \neq\{\}$. This means that if banks borrowed $R O=c L$, for all $i^{I B \bar{M}} \in I$ the expected marginal revenues of borrowing from the refinancing operations would be higher than the expected marginal costs. Consequently, $R O^{* I I}>c L^{* I I}$. Banks start to hold positive precautionary liquidity until the FOC for optimal borrowing from the refinancing operations with an active interbank market (22) with $i^{I B M * I I}=\underline{i^{I B M}}$ and $\bar{t}^{* I I}>0$ is fulfilled.
} 
banks expect a deficit to be more expensive than a surplus so that they start to hold positive precautionary liquidity, $R O^{* I I}>c L^{* I I} \cdot{ }^{34} \mathrm{~A}$ similar story can be told for $G(0)>0.5$. Then, $\bar{\gamma}=\bar{\gamma}_{u}$, and if $\bar{\gamma}=\bar{\gamma}_{u}<\gamma \leq \overline{\bar{\gamma}}$, banks will start to hold negative precautionary liquidity, $R O^{* I I}<c L^{* I I}$

In our discussion on monetary policy implementation in Section 6 , the FOCs for optimal holdings of precautionary liquidity play an important role. Considering (12) and $i^{I B M * I I}$ being equal to $\underline{i^{I B M}}$, the FOC for optimal holdings of positive precautionary liquidity becomes

$$
\left(i^{R O}-i^{D F}\right) G\left(\bar{t}^{* I I}\right)=\left(i^{D F}+2 \gamma-i^{R O}\right)\left(1-G\left(\bar{t}^{* I I}\right)\right), \quad \text { with } \bar{t}^{* I I}>0 .
$$

The expected marginal costs of holding positive precautionary liquidity in the form of interest costs are given by the LHS of (24). The RHS shows the expected marginal revenues in the form of avoided illiquidity costs. ${ }^{35}$ Analogously, the FOC for holding optimal negative precautionary liquidity is given by

$$
\left(i^{R O}-i^{L F}+2 \gamma\right) G\left(\bar{t}^{* I I}\right)=\left(i^{L F}-i^{R O}\right)\left(1-G\left(\bar{t}^{* I I}\right)\right), \text { with } \bar{t}^{* I I}<0 .
$$

The expected marginal costs of holding negative precautionary liquidity in the form of higher interest costs in case a deficit occurs, are presented by the RHS of (25). The LHS shows expected marginal revenues in the form of avoided surplus costs. ${ }^{36}$

It follows from Lemma 3 that for $\bar{\gamma}<\gamma \leq \overline{\bar{\gamma}}$ the expected marginal uncertainty costs of granting loans become

$$
\phi\left(\bar{t}^{* I I}\right) 2 \gamma \quad \text { where } \quad \phi\left(\bar{t}^{*}\right)=(1-c) \chi \int_{\bar{t}^{* I I}}^{t^{\max }} t_{b} g\left(t_{b}\right) d t_{b} .
$$

Again positive transaction costs put a wedge between the costs and revenues of using the interbank market. ${ }^{37}$ Expected marginal uncertainty costs of granting loans are thus positive and increase in $\gamma$. However, the more precautionary liquidity banks hold, the lower the costs will be (see Section 4.3 for details).

\footnotetext{
${ }^{34}$ Formally, the same arguments as made in footnote 33 hold.

${ }^{35}$ Holding positive precautionary liquidity allows banks to finance a possible deficit at the rate of $i^{R O}$ instead of $i^{I B M * I I}+\gamma=i^{D F}+2 \gamma$ which is strictly larger than $i^{R O}$ for $\gamma>\bar{\gamma}_{l}$.

${ }^{36}$ Surplus costs accrue because banks facing a liquidity surplus at the beginning of the second stage, have borrowed this liquidity at the rate of $i^{R O}$ and receive $i^{I B M * I I}-\gamma=$ $i^{L F}-2 \gamma<i^{R O}$ for $\gamma>\bar{\gamma}_{u}$ when lending it to the interbank market.

${ }^{37}$ If $\bar{t}^{* I I}>0$, the interbank rate will be at its lower bound so that the marginal costs of balancing a deficit are $i^{D F}+2 \gamma$, whereas the marginal revenues of balancing a surplus are $i^{D F}$. If $\bar{t}^{* I I}<0$, the interbank rate will be at its upper bound so that the marginal costs of balancing a deficit are $i^{L F}$, whereas marginal revenues of balancing a surplus are $i^{L F}-2 \gamma$.
} 


\subsubsection{Equilibrium III: Prohibitively High Transaction Costs}

Proposition 4 If $\gamma>\overline{\bar{\gamma}}$, the interbank market will break down and banks only use the central bank's facilities to balance their liquidity position at the second stage. Depending on the relative asymmetries of the distribution of $\tilde{t}_{b}$ and of the interest corridor, banks may hold positive, negative or no precautionary liquidity: ${ }^{38}$

$$
R O^{* I I I}\left\{\begin{array}{l}
>c L^{* I I I} \Rightarrow \bar{t}^{* I I I}=\frac{R O^{* I I I}-c L^{* I I I}}{(1-c) \chi L^{* I I I}}>0 \text { if } G(0)<\frac{i^{L F}-i^{R O}}{i^{L F}-i^{D F}}, \\
=c L^{* I I I} \Rightarrow \bar{t}^{* I I I}=\frac{R O^{* I I I}-c L^{* I I I}}{(1-c) \chi L^{* I I I}}=0 \text { if } G(0)=\frac{i^{L F}-i^{R O}}{i^{L F}-i^{D F}}, \\
<c L^{* I I I} \Rightarrow \bar{t}^{* I I I}=\frac{R O^{* I I I}-c L^{* I I I}}{(1-c) \chi L^{* I I I}}<0 \text { if } G(0)>\frac{i^{L F}-i^{R O}}{i^{L F}-i^{D F}} .
\end{array}\right.
$$

If $\gamma>\overline{\bar{\gamma}}$, interbank market transaction costs will be prohibitively high, so that all banks will exclusively use the central bank's facilities to balance their liquidity position at the second stage. Whether they hold precautionary liquidity depends again on the asymmetries of the distribution of $\tilde{t}_{b}$ and of the interest corridor. According to (11), for $R O=c L$ the expected marginal costs of a deficit are $(1-G(0))\left(i^{L F}-i^{R O}\right)$, of a surplus these costs are $G(0)\left(i^{R O}-i^{D F}\right)$. Consequently, an asymmetric distribution $(G(0) \neq 0.5)$ or an asymmetric interest corridor $\left(i^{L F}-i^{R O} \neq i^{R O}-i^{D F}\right)$ result in a deficit or a surplus that is more expensive, so that banks hold precautionary liquidity.

Referring to (12) the FOC for holding precautionary liquidity in this equilibrium reads

$$
\left(i^{R O}-i^{D F}\right) G\left(\bar{t}^{* I I I}\right)=\left(i^{L F}-i^{R O}\right)\left(1-G\left(\bar{t}^{* I I I}\right)\right) \text { with } \bar{t}^{* I I I} \gtreqless 0 .
$$

If banks hold positive precautionary liquidity $\left(\bar{t}^{* I I I}>0\right)$, the LHS of $(27)$ presents the expected marginal costs of holding this liquidity in the form of interest costs. The RHS shows the expected marginal revenues in the form of avoided illiquidity costs. If banks hold negative precautionary liquidity $\left(\bar{t}^{* I I I}<0\right)$, the LHS will present the expected marginal revenues (avoided surplus costs), whereas the RHS will show the expected marginal costs (interest costs).

Referring to Lemma 3, if the interbank market breaks down, the expected marginal uncertainty costs of granting loans will be given by

$$
\phi\left(\bar{t}^{* I I I}\right)\left(i^{L F}-i^{D F}\right), \quad \text { where } \quad \phi\left(\bar{t}^{* I I I}\right)=(1-c) \chi \int_{\bar{t}^{* I I I}}^{t^{\max }} t_{b} g\left(t_{b}\right) d t_{b} .
$$

These costs increase in the width of the interest corridor, and this increase will be less pronounced the more precautionary liquidity banks hold.

\footnotetext{
${ }^{38}$ Note again that if banks hold negative precautionary liquidity and if the negativityconstraint on $R O_{b}$ binds, $R O^{* I I I}=0$ and $\bar{t}^{* I I I}=\underline{t}=-c /(1-c) \chi<0$ (see proof of Lemma 1 in the appendix).
} 


\section{Monetary Policy and Bank Loan Supply}

With respect to the influence of monetary policy on bank loan supply, the banks' expected marginal liquidity costs of granting loans play a crucial role. In the following, we will first analyze how interbank market transaction costs and banks' uncertainty about their idiosyncratic liquidity needs affect bank loan supply. Then, we will examine the impact of different monetary policy impulses on bank loan supply, explicitly pointing to the importance of interbank market transaction costs and of banks' uncertainty over their idiosyncratic liquidity needs.

\subsection{Frictions and Uncertainty}

Result 1 In the three possible equilibria I, II, and III, the effect of a change in interbank market transaction costs on bank loan supply is

$$
\begin{aligned}
& \frac{\partial L^{* I}}{\partial \gamma}=\frac{-2 \phi(0)}{\lambda}<0, \\
& \frac{\partial L^{* I I}}{\partial \gamma}=\left\{\begin{array}{ll}
-\frac{2 \phi\left(\bar{t}^{* I I}\right)}{\lambda}+\frac{2(1-c) \chi \bar{t}^{* I I}\left(1-G\left(\bar{t}^{* I I}\right)\right)}{\lambda} & \text { if } \bar{t}^{* I I}>0 \\
-\frac{2 \phi\left(\bar{t}^{* I I}\right)}{\lambda}-\frac{2(1-c) \chi \bar{t}^{* I I} G\left(\bar{t}^{* I I}\right)}{\lambda} & \text { if } \bar{t}^{* I I}<0
\end{array}\right\}<0, \\
& \frac{\partial L^{* I I I}}{\partial \gamma}=0 .
\end{aligned}
$$

Proof See appendix.

Result 1 shows that in Equilibria I and II, increasing interbank market transaction costs have a contractionary effect on bank loan supply. In both equilibria, banks expect to use the interbank market to balance uncertain liquidity needs, so that higher transaction costs increase the expected marginal uncertainty costs of granting loans. Holdings of precautionary liquidity reduce the expected marginal uncertainty costs (see (15)). As higher interbank market transaction costs induce banks to hold more precautionary liquidity (in absolute terms), ${ }^{39}$ in Equilibrium II, the contractionary effect is weakened, as is formally shown by the second term of $\partial L^{* I I} / \partial \gamma \cdot{ }^{40}$ In Equilibrium III, the interbank market is not (expected to be) used, so that $\gamma$ has no influence on bank loan supply.

\footnotetext{
${ }^{39}$ Formally, this is revealed by the FOCs $(24)$ and (25).

${ }^{40}$ If the non-negativity constraint on $R O_{b}$ binds, banks cannot increase their negative precautionary liquidity holdings. In this case, the described weakening effect in Equilibrium II is omitted. Furthermore, $\phi\left(\bar{t}^{* I I}\right)=\phi(\underline{t})$ with $\underline{t}=-c /(1-c) \chi<0$ (see proof of Lemma 1 in the appendix).
} 
Result 2 In the three possible equilibria I, II, and III, the effect of a change in the extent of uncertainty on bank loan supply is

$$
\begin{aligned}
& \frac{\partial L^{* I}}{\partial \chi}=\frac{-\phi(0) 2 \gamma}{\lambda \chi}<0, \\
& \frac{\partial L^{* I I}}{\partial \chi}=\frac{-\phi\left(\bar{t}^{* I I}\right) 2 \gamma}{\lambda \chi}<0, \\
& \frac{\partial L^{* I I I}}{\partial \chi}=\frac{-\phi\left(\bar{t}^{* I I I}\right)\left(i^{L F}-i^{D F}\right)}{\lambda \chi}<0 .
\end{aligned}
$$

Proof See appendix.

Result 2 reveals that in all equilibria, an increase in the extent of uncertainty leads to a decrease in bank loan supply. Higher uncertainty, here in the form of a higher dispersion of $\chi \tilde{t}_{b}$, means that banks expect a larger deficit as well as a larger surplus. Consequently, the expected marginal uncertainty costs increase and banks reduce their loan supply. In Equilibria II and III, this effect is mitigated as the increase in $\chi$ implies that banks hold more precautionary liquidity, which has a negative effect on expected marginal uncertainty costs (see $(15))^{41}$

\subsection{Equal Change in All Central Bank Interest Rates}

Starting our analysis of the impact of different monetary policy impulses on bank loan supply with an equal change in all central bank's interest rates, we get

Result 3 If $\frac{d i^{D F}}{d i^{R O}}=\frac{d i^{L F}}{d i^{R O}}=1$, we obtain

$$
\frac{\partial L^{* j}}{\partial i^{R O}}=-\frac{c}{\lambda}<0 \quad \text { and } \quad \frac{\partial^{2} L^{* j}}{\partial i^{R O} \partial \chi}=\frac{\partial^{2} L^{* j}}{\partial i^{R O} \partial \gamma}=0 \quad \forall j .
$$

Proof See appendix.

An equal decrease in all central bank interest rates has the same expansionary effect on bank loan supply in all equilibria $j=I, I I, I I I$. The crucial point is that this monetary policy impulse only leads to a change in certain

\footnotetext{
${ }^{41}$ If the non-negativity constraint on $R O_{b}$ binds, banks will not be able to increase their holdings of negative precautionary liquidity. The effect of an increase in $\chi$ on $L^{* I I}$ and $L^{* I I I}$ will thus be stronger. Formally, we have

$$
\begin{aligned}
& \frac{\partial L^{* I I}}{\partial \chi}=\frac{-\phi(\underline{t}) 2 \gamma}{\lambda \chi}+\frac{2 \gamma \underline{t} g(\underline{t}) c}{\lambda \chi}<0, \\
& \frac{\partial L^{* I I I}}{\partial \chi}=\frac{-\phi(\underline{t})\left(i^{L F}-i^{D F}\right)}{\lambda \chi}+\frac{\left(i^{L F}-i^{D F}\right) \underline{t} g(\underline{t}) c}{\lambda \chi}<0,
\end{aligned}
$$

with $\underline{t}=-c /(1-c) \chi<0$ (see proof of Lemma 1 in the appendix).
} 
marginal liquidity costs, whereas the expected marginal uncertainty costs of granting loans remain unchanged. It follows from (23), (26), and (28) that this monetary policy impulse would only have an impact on these costs if banks changed their holdings of precautionary liquidity, i.e., if $\bar{t}^{*}$ changed. However, an equal change in all interest rates has no impact on the marginal costs and revenues of holding precautionary liquidity, as revealed by the FOCs (24), (25), and (27). As $\gamma$ and $\chi$ are only relevant for expected marginal uncertainty costs of granting loans, neither the interbank market friction in the form of transaction costs nor the uncertainty about idiosyncratic liquidity needs has an impact on the effectiveness of this monetary policy impulse on bank loan supply.

\subsection{Change in the Width of the Interest Corridor}

As an alternative to an equal change in all central bank interest rates, the central bank may change the width of the interest corridor around its policy rate. This leads us to

Result 4 If $\frac{d i^{D F}}{d i^{L F}}=-1$, we obtain

$$
\begin{array}{ll}
\frac{\partial L^{* I}}{\partial i^{L F}}=0, & \frac{\partial L^{* I I}}{\partial i^{L F}}=-\frac{(1-c) \chi||^{* I I} \mid}{\lambda}<0, \frac{\partial L^{* I I I}}{\partial i^{L F}}=-\frac{2 \phi\left(\bar{t}^{* I I I}\right)}{\lambda}<0, \\
\frac{\partial^{2} L^{* I}}{\partial i^{L F} \partial \chi}=0, & \frac{\partial^{2} L^{* I I}}{\partial i^{L F} \partial \chi}=-\frac{(1-c)\left|\vec{t}^{* I I}\right|}{\lambda}<0, \frac{\partial^{2} L^{* I I I}}{\partial i^{L F} \partial \chi}=-\frac{2 \phi\left(\bar{t}^{* I I I}\right)}{\lambda \chi}<0 \\
\frac{\partial^{2} L^{* I}}{\partial i^{L F} \partial \gamma}=0, & \frac{\partial^{2} L^{* I I}}{\partial i^{L F} \partial \gamma}=\left\{\begin{array}{ll}
-\frac{(1-c) \chi\left(1-G\left(\bar{t}^{* I I}\right)\right)}{\lambda \gamma g\left(\bar{t}^{* I I}\right)} & \text { if } \bar{t}^{* I I}>0 \\
-\frac{(1-c) \chi G\left(\bar{*}^{* I I}\right)}{\lambda \gamma g\left(\bar{t}^{* I I}\right)} & \text { if } \bar{t}^{* I I}<0
\end{array}\right\}<0, \frac{\partial^{2} L^{* I I I}}{\partial i^{L F} \partial \gamma}=0 .
\end{array}
$$

Proof See appendix.

In our model, the symmetry/asymmetry of the corridor refers to the refinancing rate $i^{R O}$. The corridor will be symmetric (asymmetric) if this rate is (not) the mid-point of the corridor. ${ }^{42}$ By changing the width of a symmetric interest corridor, the central bank may influence bank loan supply without changing its policy rate $i^{R O}$. As $i^{R O}$ remains unchanged, certain marginal liquidity costs of granting loans do not alter. A potential effect of this monetary policy impulse on bank loan supply must thus be due to a change in expected marginal uncertainty costs. In Equilibrium I, banks never use the facilities to balance their uncertain liquidity needs. Changing the width of the corridor has therefore no effect on their loan supply decision. However, in Equilibria II and III, banks expect to use at least one of the facilities. In these equilibria widening the interest corridor has a contractionary effect on bank loan supply.

\footnotetext{
${ }^{42}$ In the literature, the symmetry/asymmetry of the interest corridor also refers to the central bank's target interbank rate (Bindseil, 2014, chapters 4 and 13). With persisting high levels of excess liquidity the target rate is the deposit facility rate and, independent of the position of the refinancing rate, the interest corridor is considered to be asymmetric.
} 
In Equilibrium II, an increase in the corridor width leads to higher marginal costs of holding positive as well as negative precautionary liquidity, see FOCs (24) and (25), so that banks reduce their holdings of this liquidity. This increases the expected marginal uncertainty costs of granting loans, and banks reduce their loan supply. ${ }^{43}$ Due to the reduced holdings of precautionary liquidity banks expect to use the interbank market more intensively. An increase in $\gamma$ hence amplifies the contractionary effect of a corridor widening on bank loan supply. Furthermore, the reduced holdings of precautionary liquidity are more significant, the higher the extent of uncertainty is, so that the effect of this monetary policy impulse on bank loan supply increases in $\chi$.

In Equilibrium III, banks do not adjust their precautionary liquidity holdings relative to their loan supply, i.e., $\bar{t}^{* I I I}$ remains unchanged, as the symmetry of the interest corridor implies for all possible facility rates $G\left(\bar{t}^{*}\right)=$ $1-G\left(\bar{t}^{*}\right)=0.5$. Only in this case, the expected marginal costs equal the expected marginal revenues of holding precautionary liquidity, see FOC (27). Consequently, if the corridor is widened, the expected marginal uncertainty costs of granting loans will change only because of the marginal uncertainty costs $\left(i^{L F}-i^{D F}\right)$ will increase. As the expected deficit and the expected surplus increase in the extent of uncertainty, the weight of these costs $\phi\left(\bar{t}^{* I I I}\right)$ becomes larger, so that the impact of this monetary policy impulse on bank loan supply also increases in the extent of uncertainty.

\subsection{Change in the Asymmetry of the Interest Corridor}

There are several ways to implement an asymmetric interest corridor or to change an already existing asymmetry of the corridor. Here, we take a closer look at the consequences of a sole change in the deposit rate $i^{D F}$. Building on this analysis, we will draw some general conclusions with respect to the consequences of a change in the asymmetry of the interest corridor on bank loan supply at the end of this section. Changing only $i^{D F}$ leads to

\footnotetext{
${ }^{43}$ If the non-negativity constraint on $R O_{b}$ binds, banks will not be able to change their holdings of negative precautionary liquidity. The monetary policy impulse will have no effect on bank loan supply.
} 
Result 5 Changing only the deposit rate $i^{D F}\left(d i^{R O}=d i^{L F}=0\right)$, we obtain

$$
\begin{array}{ll}
\frac{\partial L^{* I}}{\partial i^{D F}}=0, & \frac{\partial L^{* I I}}{\partial i^{D F}}=\left\{\begin{array}{ll}
\frac{(1-c) \chi \bar{t}^{* I I}}{\lambda}>0 & \text { if } \bar{t}^{* I I}>0 \\
\text { if } \bar{t}^{* I I}<0
\end{array},\right. \\
\frac{\partial L^{* I I I}}{\partial i^{D F}}+\frac{\phi\left(\bar{t}^{* I I I}\right)}{\lambda} \frac{(1-c) \chi \bar{t}^{* I I I} G\left(\bar{t}^{* I I I}\right)}{\lambda}>0, & \frac{\partial^{2} L^{* I I}}{\partial i^{D F} \partial \chi}\left\{\begin{array}{ll}
=\frac{(1-c))^{* I I}}{\lambda}>0 & \text { if } \bar{t}^{* I I}>0 \\
=0 & \text { if } \bar{t}^{* I I}<0
\end{array},\right. \\
\frac{\partial^{2} L^{* I}}{\partial i^{D F} \partial \chi}=0, & \frac{\partial^{2} L^{* I I}}{\partial i^{D F} \partial \gamma} \begin{cases}=\frac{(1-c) \chi G\left(\bar{t}^{* I I}\right)}{\lambda \gamma g\left(\bar{t}^{* I I}\right)}>0 & \text { if } \bar{t}^{* I I}>0 \\
=0 & \text { if } \bar{t}^{* I I}<0\end{cases} \\
\frac{\partial^{2} L^{* I I I}=\frac{\phi\left(\bar{t}^{* I I I}\right)}{\lambda \chi}}{\partial i^{D F} \partial \chi}+\frac{(1-c) \chi \bar{t}^{* I I I} G\left(\bar{t}^{* I I I}\right)}{\lambda \chi}>0, & \\
\frac{\partial^{2} L^{* I}}{\partial i^{D F} \partial \gamma}=0, & \\
\frac{\partial^{2} L^{* I I I}}{\partial i^{D F} \partial \gamma}=0 . &
\end{array}
$$

Proof See appendix.

A decrease in $i^{D F}$ has either no or a contractionary effect on bank loan supply. In Equilibrium I and in Equilibrium II for $\bar{t}^{* I I}<0$, banks do not (expect to) use the deposit facility. A change in $i^{D F}$ therefore has no effect on bank loan supply.

If banks hold positive precautionary liquidity in Equilibrium II $\left(\bar{t}^{* I I}>0\right)$, a decrease in $i^{D F}$ will have a contractionary effect on bank loan supply. As holding positive precautionary liquidity will become more expensive, see FOC (24), banks will reduce their holdings of this liquidity. The expected marginal uncertainty costs of granting loans will increase, and banks will reduce their loan supply. Analogous to the monetary policy impulse of a widening of the interest corridor, this policy impulse is more pronounced the higher the interbank market transaction costs and the extent of uncertainty are.

In Equilibrium III, a decrease in $i^{D F}$ has a contractionary effect on bank loan supply. The driving force is the increase in the marginal uncertainty costs of granting loans $\left(i^{L F}-i^{D F}\right)$. This direct effect is reflected by the first term of $\partial L^{* I I I} / \partial i^{D F}$. If banks hold positive precautionary liquidity $\left(\bar{t}^{*}>0\right)$, this contractionary effect will be reinforced. The decrease in $i^{D F}$ increases the expected marginal costs of holding positive precautionary liquidity, so that banks will reduce their holdings of this liquidity. The resulting increase in the expected marginal uncertainty costs has a contractionary effect on bank loan supply (see second term of $\left.\partial L^{I I I *} / \partial i^{D F}\right)$. If banks hold negative precautionary liquidity $\left(\bar{t}^{*}<0\right)$ or no precautionary liquidity $\left(\bar{t}^{*}=0\right)$, the contractionary effect will be weakened as the expected marginal revenues of holding this liquidity will become higher so that banks will increase or start to hold negative precautionary liquidity which would have a negative effect on the expected marginal 
uncertainty costs of granting loans. ${ }^{44}$ The direct effect of this monetary policy impulse on the expected marginal uncertainty costs (increase in $i^{L F}-i^{D F}$ ) is stronger than the indirect effect (change in holdings of precautionary liquidity). As marginal uncertainty costs are more important to banks the higher the extent of uncertainty is (formally, $\phi\left(\bar{t}^{*}\right)$ increases in $\chi$ ), the effect of this policy impulse is more pronounced the higher the extent of uncertainty is.

From this analysis we can conclude the following general results with respect to the effect of a change in the asymmetry of the interest corridor on bank loan supply. In Equilibrium I, changes in the asymmetry of the corridor do not have an impact on bank loan supply. In Equilibrium II, changes in the asymmetry of the corridor resulting in an increase in the costs of holding precautionary liquidity have a negative effect on bank loan supply. In Equilibrium III, those changes in the asymmetry of the corridor which leads to an increase in the spread between $i^{L F}$ and $i^{D F}$ have a negative effect on bank loan supply. Precautionary holdings of liquidity reinforce or dampen this effect.

\subsection{Collateralization and Minimum Reserve Requirements}

In our model, we have neglected two main elements of the ECB's operational framework: the collateralization of central bank credits and the minimum reserve requirements. Considering these elements is not crucial for our analysis as they only change our results quantitatively.

If we took the collateralization of central bank credits into consideration, banks would face opportunity costs of holding collateral. As a result, the expected marginal liquidity costs would increase ${ }^{45}$ Bank loan supply would be lower but our model results would not change qualitatively.

Reserve requirements lead to a structural liquidity deficit of the banking sector. In our model, such a deficit is already captured by considering cash withdrawals. Introducing reserve requirements would therefore simply increase the existing structural deficit. A main feature of the Eurosystem's minimum reserve system is that banks can make use of the averaging provision of required reserves during the reserve maintenance period. This allows banks to smooth out liquidity fluctuations. In our model, the marginal uncertainty costs of granting loans would decrease. Although this would have a positive effect on bank loan supply, the qualitative results of our model would not change.

\footnotetext{
${ }^{44}$ This weakening effect will not exist if the non-negativity constraint on $R O_{b}$ is binding, as then, banks would not be able to increase their negative holdings of precautionary liquidity any further. Formally, we have in this case $\partial L^{* I I I} / \partial i^{D F}=\phi(\underline{t}) / \lambda>0$ and $\partial^{2} L^{* I I I} / \partial i^{D F} \partial \chi=(\phi(\underline{t})-\underline{t} g(\underline{t}) c) / \lambda \chi>0$, with $\underline{t}=-c /((1-c) \chi)<0$ (see proof of Lemma 1 in the appendix).

${ }^{45}$ For a respective analysis see, for example, Neyer and Wiemers (2004) and Berentsen and Monnet (2008).
} 


\section{Conclusion}

The interbank market plays a crucial role for monetary policy implementation as it serves as the starting point of the monetary policy transmission mechanism. Based on a theoretical model, this paper analyzes how far interbank market frictions in the form of broadly defined transaction costs may impede the transmission mechanism. We find that interbank market frictions have a negative impact on bank loan supply but that they are not an impediment for the monetary transmission mechanism. The central bank can still influence commercial banks' expected liquidity costs and thus their loan supply. First, the central bank's standing facilities offer an alternative to using the frictionburdened and thus costly interbank market. Second, and this is a main result of our paper, the rates on the facilities determine the costs of friction-induced holdings of precautionary liquidity. Increasing the rate on the deposit facility and decreasing the rate on the lending facility are expansionary monetary policy measures. Note that the sign of the central bank's interest rates does not play a role for this result.

Our model allows us to draw some conclusions for bank loan supply in the euro area and the ECB's monetary policy. During the financial crisis, interbank market frictions became stronger which made interbank trading more expensive. Furthermore, bank idiosyncratic liquidity risk increased. Both led to a rise in banks' expected liquidity costs which had a negative impact on bank loan supply. However, from October 2008, when the collapse of the investment bank Lehman Brothers marked a peak of the global financial crisis, up to now (October 2018) the ECB has not only decreased its main refinancing rate from $4,75 \%$ to a historically low level of $0 \%$, but it has also narrowed the interest corridor of its standing facilities from 200 basis points to 65 basis points. Thus, the ECB has not only significantly reduced banks' expected liquidity costs by lowering the price for liquidity borrowed directly from the central bank, but in addition, it has reduced interbank market friction-induced liquidity costs. Consequently, monetary policy has not only been extremely expansive, but it has also made sure that interbank market frictions have not been a significant impediment for its pass-through to bank loan supply. Nevertheless, bank lending in the euro area, especially in the periphery, has been relatively weak. This indicates that there have been structural problems, such as insufficient bank capital or the lack of competitive projects in need of financing.

Finally, it should be noted that in our model, the banking sector as a whole faces a structural liquidity deficit forcing banks to borrow liquidity from the central bank. As the central bank totally satisfies banks' demand for liquidity, excess liquidity holdings are purely demand-driven. However, the strongly increasing aggregate excess liquidity holdings, which can be observed in the euro area since 2015, are mainly due to the Eurosystem's large-scale asset purchases under its Expanded Asset Purchase Programme (APP) and are thus mainly supply-driven (see Baldo et al, 2017). These asset purchases even implied that since August 2015 the banking sector as a whole has faced a structural liquidity 
surplus. ${ }^{46}$ However, despite this surplus there still have been banks borrowing liquidity from the Eurosystem. A deep analysis of commercial banks' liquidity management and the consequences for bank loan supply and monetary policy implementation in an environment of a structural liquidity surplus and a heterogeneous banking sector thus presents an interesting issue for future research.

\section{A Appendix}

\section{A.1 Proof of Lemma 1}

It has to be shown that if the non-negativity constraint on $R O_{b}$ does not bind, optimal borrowing from the central bank's refinancing operation will be described by (8). It follows from (3) to (5), that the first stage optimization problem of bank $b$ reads:

$$
\begin{aligned}
\max _{L_{b} \in \mathbb{R}_{0}^{+}, R O_{b} \in \mathbb{R}_{0}^{+}} f\left(L_{b}, R O_{b}\right) & =i^{L} L_{b}-\frac{1}{2} \lambda L_{b}^{2}-i^{R O} R O_{b} \\
& -\max \left\{i^{I B M}-\gamma, i^{D F}\right\} \int_{t^{\text {min }}}^{\bar{t}_{b}} N_{b} g\left(t_{b}\right) d t_{b} \\
& -\min \left\{i^{I B M}+\gamma, i^{L F}\right\} \int_{\bar{t}_{b}}^{t^{\text {max }}} N_{b} g\left(t_{b}\right) d t_{b},
\end{aligned}
$$

with $N_{b}$ given by (6) and $\bar{t}_{b}$ by (7).

By applying Leibniz's rule and making use of the fact that $N_{b}=0$ for $t_{b}=\bar{t}_{b}$, we obtain:

$$
\begin{aligned}
f_{R O_{b}}^{\prime}\left(L_{b}, R_{b}\right) & =-i^{R O}-\max \left\{i^{I B M}-\gamma, i^{D F}\right\} \int_{t^{\min }}^{\bar{t}_{b}} \frac{\partial N_{b}}{\partial R O_{b}} g\left(t_{b}\right) d t_{b} \\
& -\min \left\{i^{I B M}+\gamma, i^{L F}\right\} \int_{\bar{t}_{b}}^{t^{\max }} \frac{\partial N_{b}}{\partial R O_{b}} g\left(t_{b}\right) d t_{b} .
\end{aligned}
$$

We can infer from (6) that $\frac{\partial N_{b}}{\partial R O_{b}}=-1$. Insertion of this in (30) and rewriting terms yields

$$
\begin{aligned}
f_{R O_{b}}^{\prime}\left(L_{b}, R_{b}\right) & =-i^{R O}+\max \left\{i^{I B M}-\gamma, i^{D F}\right\} G\left(\bar{t}_{b}\right) \\
& +\min \left\{i^{I B M}+\gamma, i^{L F}\right\}\left[1-G\left(\bar{t}_{b}\right)\right] .
\end{aligned}
$$

Note that $f_{R O_{b}}^{\prime}\left(L_{b}, R_{b}\right)$ decreases in $G\left(\bar{t}_{b}\right) \in[0,1]$, which in turn (weakly) increases in $\bar{t}_{b}$. Moreover, we know from (7) that

$-\bar{t}_{b}$ increases in $R O_{b}$, so that $f_{R O_{b}}^{\prime}\left(L_{b}, R_{b}\right)$ (weakly) decreases in $R O_{b}$,

- and from the restriction $R O_{b} \geq 0$ that $\bar{t}_{b}$ is restricted to $\bar{t}_{b} \geq-\frac{c}{(1-c) \chi}=: \underline{t}$.

Denoting optima by the superscript opt, we can distinguish three cases:

1. If $i^{I B M}>i^{R O}+\gamma$, then $f_{R O_{b}}^{\prime}\left(L_{b}, R_{b}\right)>0$ for all $R O_{b}<\infty$. Therefore, we obtain $R O_{b}^{\text {opt }} \rightarrow \infty$.

${ }^{46}$ Note that since the beginning of the ECB's full allotment policy in 2008, the banking sector operates in an environment of excess liquidity. However, the difference between the period until August 2015 and the period thereafter is that since August 2015, the banking sector has no longer been able to reduce excess liquidity by decreasing its borrowing from the ECB. Even if no bank borrows from the ECB, there will be excess liquidity. 
2. If $i^{I B M} \in\left[i^{R O}-\gamma, i^{R O}+\gamma\right]$, the first order conditions for optimal borrowing from the refinancing operations are

$$
f_{R O_{b}}^{\prime}\left(L_{b}^{o p t}, R_{b}^{o p t}\right) \leq 0, \quad f_{R O_{b}}^{\prime}\left(L_{b}^{o p t}, R_{b}^{o p t}\right) \cdot R O_{b}^{o p t}=0, \quad R O_{b}^{o p t} \geq 0 .
$$

Accordingly, if the non-negativity constraint for $R O_{b}$ does not bind, marginal costs and expected marginal revenues of borrowing from the refinancing operations will be balanced, $f_{R O_{b}}^{\prime}\left(L_{b}^{o p t}, R_{b}^{o p t}\right)=0$.

However, if for $R O_{b}=0$, i.e. for $\bar{t}_{b}=\underline{t}$, expected marginal revenues are strictly lower than marginal costs, the non-negativity constraint becomes binding, further decreases in $R O_{b}$ are not possible. Then, $R O_{b}^{o p t}=0$ and $f_{R O_{b}}^{\prime}\left(L_{b}^{o p t}, R_{b}^{o p t}\right)<0$.

3. If $i^{I B M}<i^{R O}-\gamma$, then $f_{R O_{b}}^{\prime}\left(L_{b}, R_{b}\right)<0$ for all $R O_{b} \geq 0$. The non-negativity constraint on $R O_{b}$ binds, so that $R O_{b}^{o p t}=0$.

An interbank rate $i^{I B M} \notin\left[i^{R O}-\gamma, i^{R O}+\gamma\right]$ is not a possible equilibrium rate: Such an interbank rate would imply that $R O_{b}^{\text {opt }}=0$ or $R O_{b}^{\text {opt }} \rightarrow \infty$, respectively. At the first stage, all banks are identical, i.e. they solve the same optimization problem and $R O_{b}^{o p t}$ is the same for all banks. Therefore, $R O_{b}^{o p t}=0$ or $R O_{b}^{o p t} \rightarrow \infty$ would imply that also at the aggregate level borrowing from the refinancing operations would be zero or infinite so that aggregate supply or demand in the interbank market at the second stage would be zero, so that there would not be an active interbank market. Accordingly, the cases 1 and 3 cannot occur in equilibrium. For the sake of a clearer presentation we thus exclude these non-relevant cases already at this stage. In the remaining case 2, optimal borrowing from the refinancing operations requires $f_{R O_{b}}^{\prime}\left(L_{b}^{o p t}, R_{b}^{o p t}\right)=0$ if the non-negativity constraint for $R O_{b}$ does not bind. Setting thus (31) equal to zero and solving for $i^{R O}$ gives (8). Consequently, we have shown that if the non-negativity constraint for $R O_{b}$ does not bind, a bank's optimal borrowing from the refinancing operations will be described by (8).

\section{A.2 Proof of Lemma 3}

It has to be shown that if the non-negativity constraint on $R O_{b}$ does not bind, optimal loan supply to the non-banking sector will be described by (13). The respective optimization problem of bank $b$ is given by (29). By applying Leibniz's rule on (29) and making use of the property that $N_{b}=0$ for $t_{b}=\bar{t}_{b}$, we obtain:

$$
\begin{aligned}
f_{L_{b}}^{\prime}\left(L_{b}, R_{b}\right) & =i^{L}-\lambda L_{b}-\max \left\{i^{I B M}-\gamma, i^{D F}\right\} \int_{t^{m i n}}^{\bar{t}_{b}} \frac{\partial N_{b}}{\partial L_{b}} g\left(t_{b}\right) d t_{b} \\
& -\min \left\{i^{I B M}+\gamma, i^{L F}\right\} \int_{\bar{t}_{b}}^{t^{\max }} \frac{\partial N_{b}}{\partial L_{b}} g\left(t_{b}\right) d t_{b} .
\end{aligned}
$$

We can infer from (6) that $\frac{\partial N_{b}}{\partial L_{b}}=c+(1-c) \chi t_{b}$. Insertion of this in (33) and rewriting terms yields

$$
\begin{aligned}
f_{L_{b}}^{\prime}\left(L_{b}, R_{b}\right) & =i^{L}-\lambda L_{b} \\
& -c\left[\max \left\{i^{I B M}-\gamma, i^{D F}\right\} G\left(\bar{t}_{b}\right)+\min \left\{i^{I B M}+\gamma, i^{L F}\right\}\left[1-G\left(\bar{t}_{b}\right)\right]\right] \\
& -(1-c) \chi \max \left\{i^{I B M}-\gamma, i^{D F}\right\} \int_{t^{\min }}^{\bar{t}_{b}} t_{b} g\left(t_{b}\right) d t_{b} \\
& -(1-c) \chi \min \left\{i^{I B M}+\gamma, i^{L F}\right\} \int_{\bar{t}_{b}}^{t^{\max }} t_{b} g\left(t_{b}\right) d t_{b} .
\end{aligned}
$$

Using the result for optimal borrowing from the refinancing operations given by (8), we can replace the term in square brackets in the second row of $(34)$ by $i^{R O}$. Due to $E\left[t_{b}\right]=0$ 
we have $-\int_{t^{\text {min }}}^{\bar{t}_{b}} t_{b} g\left(t_{b}\right) d t_{b}=\int_{\bar{t}_{b}}^{t^{\text {max }}} t_{b} g\left(t_{b}\right) d t_{b}$. This allows us to replace $-\int_{t^{\text {min }}}^{\bar{t}_{b}} t_{b} g\left(t_{b}\right) d t_{b}$ by $\int_{\bar{t}_{b}^{o p t}}^{t^{\text {max }}} t_{b} g\left(t_{b}\right) d t_{b}$ in (34). Setting then (34) equal to zero and solving for $i^{L}$ gives (13). Consequently, we have shown that if the non-negativity constraint for $R O_{b}$ does not bind (we have made use of (8) which will only hold if this restriction does not bind), a bank's optimal lending to the non-banking sector will be described by (13).

\section{A.3 Proof of Proposition 1}

(a) It has to be shown that if $\gamma>\overline{\bar{\gamma}}$, we will have an inactive interbank market, i. e. $B^{*}=0$. If $i^{I B M}+\gamma>i^{L F}$, no bank will borrow from the interbank market as borrowing from the lending facility is less costly, i.e. $B=0$. If $i^{I B M}-\gamma<i^{D F}$, no bank will lend to the interbank market as using the deposit facility earns a higher revenue, i.e. $B=0$. Consequently, there is an upper and a lower bound for $i^{I B M}$.

$$
\overline{i^{I B M}}=i^{L F}-\gamma, \quad \underline{i^{I B M}}=i^{D F}+\gamma .
$$

If $\overline{i^{I B M}}<\underline{i^{I B M}}$, there is no $i^{I B M}$ at which both market sides are willing to transact, i.e. $I=\{\}(I$ is defined by (19)). This will be the case if $\gamma>\overline{\bar{\gamma}}$ :

$$
\begin{aligned}
& \overline{i^{I B M}}=i^{L F}-\gamma<\underline{i^{I B M}}=i^{D F}+\gamma \\
& \Rightarrow \gamma>\frac{i^{L F}-i^{D F}}{2}=\overline{\bar{\gamma}} .
\end{aligned}
$$

Consequently, if $\gamma>\overline{\bar{\gamma}}, B^{*}=0$. However, for $\gamma \leq \overline{\bar{\gamma}}, I \neq\{\}$, there will an active interbank market.

(b) It has to be shown that if $\gamma \leq \overline{\bar{\gamma}}$,

$$
i^{I B M *}= \begin{cases}\frac{i^{I B M}}{\tilde{i}^{I B M}} \in I & \text { if } R O^{*}>c L^{*}, \\ \frac{\text { if } R O^{*}=c L^{*},}{i^{I B M}} & \text { if } R O^{*}<c L^{*} .\end{cases}
$$

If $\gamma \leq \overline{\bar{\gamma}}$, we have an active interbank market, and (38) presents the equilibrium interbank rate for the different possible liquidity scenarios. The first and third line of (38) are trivial. Liquidity excess, $R O^{*}>c L^{*}$, (shortage, $R O^{*}<c L^{*}$ ) will bring $i^{I B M *}$ to its lower (upper) bound. If liquidity conditions are balanced $\left(R O^{*}=c L^{*}\right), i^{I B M *}=\tilde{i}^{I B M}$ will be the only subgame perfect equilibrium interest rate: Having an active interbank market, the FOC for borrowing $R O=c L$ from the refinancing operations in the first stage given in Lemma 1 becomes

$$
\left(i^{I B M *}-\gamma\right) G(0)+\left(i^{I B M *}+\gamma\right)(1-G(0))=i^{R O} .
$$

Solving (39) for $i^{I B M *}$ results in $\tilde{i}^{I B M}$ as given in (10). Consequently, when borrowing $R O=c L, \tilde{i}^{I B M}$ is the only interbank rate at which (39) is fulfilled, at which expected marginal revenues and marginal costs are balanced. For all other $i^{I B M}$ expected marginal revenues will strictly deviate from marginal costs, inducing banks to borrow more or less than $c L$. Consequently, for $R O^{*}=c L^{*}, \tilde{i}^{I B M}$ is the only subgame perfect equilibrium interbank rate.

\section{A.4 Proof of Proposition 2}

It has to be shown that if $\gamma \leq \bar{\gamma}, R O^{*}=c L^{*}$ and $i^{I B M *}=\tilde{i}^{I B M}$, with $\bar{\gamma}$ defined in (21) and $\tilde{i}^{I B M}$ in (10). As $\bar{\gamma} \leq \overline{\bar{\gamma}}$ (see proof of Proposition 3), $\gamma \leq \bar{\gamma}$ implies that we have an 
active interbank market (see Proposition 1). Having an active interbank market, the FOC for optimal borrowing from the refinancing operations given in Lemma 1 becomes

$$
\left(i^{I B M *}-\gamma\right) G(\bar{t})+\left(i^{I B M *}+\gamma\right)(1-G(\bar{t}))=i^{R O} .
$$

When borrowing $\boldsymbol{R O}=\boldsymbol{c} \boldsymbol{L}, \bar{t}=0$ (see (7)), i.e. $G(\bar{t})$ equals $G(0)$, and the interbank rate which fulfills (40) equals $\tilde{i}^{I B M}$. To have $\tilde{i}^{I B M}=i^{I B M *}, \tilde{i}^{I B M}$ must be $\in I=\left[\underline{i^{I B M}}, \overline{i^{I B M}}\right]$ (see (19)). If $\gamma \leq \bar{\gamma}$, this will be the case:

For $\tilde{i}^{I B M} \geq \underline{i^{I B M}}$, we must have

$$
\underline{i^{I B M}}=i^{D F}+\gamma \leq \tilde{i}^{I B M}=i^{R O}-\gamma(1-2 G(0)), \Rightarrow \frac{i^{R O}-i^{D F}}{2(1-G(0))} \geq \gamma, \quad \Rightarrow \bar{\gamma}_{l} \geq \gamma .
$$

As we only look at $\gamma<\bar{\gamma}$, and $\bar{\gamma}:=\min \left\{\bar{\gamma}_{l}, \bar{\gamma}_{u}\right\}$ (see (21)), $\tilde{i}^{I B M} \geq \underline{i^{I B M}}$.

For $\tilde{i}^{I B M} \leq \overline{i^{I B M}}$, we must have

$$
\overline{i^{I B M}}=i^{L F}-\gamma \geq \tilde{i}^{I B M}=i^{R O}-\gamma(1-2 G(0)), \Rightarrow \frac{i^{L F}-i^{R O}}{2 G(0)} \geq \gamma, \Rightarrow \bar{\gamma}_{u} \geq \gamma .
$$

As we only look at $\gamma<\bar{\gamma}$, and $\bar{\gamma}:=\min \left\{\bar{\gamma}_{l}, \bar{\gamma}_{u}\right\}$ (see (21)), $\tilde{i}^{I B M} \leq \overline{i^{I B M}}$. As conditions (41) and (42) are fulfilled, $\tilde{i}^{I B M} \in I$. Consequently, $R O^{*}=c L^{*}$ and $i^{I B M *}=\tilde{i}^{I B M}$ is an equilibrium.

When borrowing $\boldsymbol{R O}>\boldsymbol{c L}, i^{I B M}=\underline{i^{I B M}}$, see Proposition 1. Inserting $\underline{i^{I B M}}=i^{D F}+\gamma$ (see (17)) into (40) and solving for $\gamma$, we get that the FOC is only fulfilled for

$$
\gamma=\frac{i^{R O}-i^{D F}}{2(1-G(\bar{t}))}
$$

However, with $R O>c L, \bar{t}>0$ (see (7)), i.e. $G(\bar{t})>G(0)$, so that

$$
\gamma=\frac{i^{R O}-i^{D F}}{2(1-G(\bar{t}))}>\frac{i^{R O}-i^{D F}}{2(1-G(0))}=\bar{\gamma}_{l} .
$$

As $\bar{\gamma}_{l} \geq \bar{\gamma}$ (see (21)), $R O>c L$ cannot be an equilibrium if $\gamma \leq \bar{\gamma}$.

When borrowing $\boldsymbol{R O}<\boldsymbol{c L}, i^{I B M}=\overline{i^{I B M}}$, see Proposition 1. Inserting $\overline{i^{I B M}}=i^{L F}-\gamma$ (see (16)) into (40) and solving for $\gamma$, we get that the FOC is only fulfilled for

$$
\gamma=\frac{i^{L F}-i^{R O}}{2 G(\bar{t})} .
$$

However, with $R O<c L, \bar{t}<0$ (see (7)), i.e. $G(\bar{t})<G(0)$, so that

$$
\gamma=\frac{i^{L F}-i^{R O}}{2 G(\bar{t})}>\frac{i^{L F}-i^{R O}}{2 G(0)}=\bar{\gamma}_{u} .
$$

As $\bar{\gamma}_{u} \geq \bar{\gamma}$ (see (21)), $R O<c L$ cannot be an equilibrium if $\gamma \leq \bar{\gamma}$

Consequently, we have shown that if $\gamma \leq \bar{\gamma}, R O^{*}=c L^{*}$ and $i^{I B M *}=\tilde{i}^{I B M}$ will be the only possible equilibrium.

\section{A.5 Proof of Proposition 3}

(a) It has to be shown that if $\bar{\gamma}=\bar{\gamma}_{l}<\gamma \leq \overline{\bar{\gamma}}, R O^{*}>c L^{*}$ and $i^{I B M *}=\underline{i^{I B M}}$. It is helpful to have a closer look at the relation between $\bar{\gamma}, \bar{\gamma}_{l}, \bar{\gamma}_{u}$, and $\overline{\bar{\gamma}}$ first. $\bar{\gamma}, \bar{\gamma}_{l}$, and $\bar{\gamma}_{u}$ are defined in $(21), \overline{\bar{\gamma}}$ in (18). Note that we assume throughout the paper that either the interest corridor or the distribution of $\tilde{t}$ is asymmetric or that both are symmetric. 
a1. If $\bar{\gamma}=\bar{\gamma}_{l}=\bar{\gamma}_{\boldsymbol{u}}$, we will have a symmetric interest corridor and a symmetric distribution of $\tilde{t}$ :

$$
\begin{aligned}
& \bar{\gamma}_{l}=\frac{i^{R O}-i^{D F}}{2(1-G(0))}=\frac{i^{L F}-i^{R O}}{2 G(0)}=\bar{\gamma}_{u} \\
& \Rightarrow \frac{i^{R O}-i^{D F}}{i^{L F}-i^{R O}}=\frac{1-G(0)}{G(0)} .
\end{aligned}
$$

If there is a symmetric interest corridor, the LHS of (48) will be equal to 1. Equality of both sides of the equation requires that also the RHS must be equal to 1, which will only be the case if there is a symmetric distribution of $\tilde{t}$. Consequently, $\bar{\gamma}_{l}=\bar{\gamma}_{u}$ implies that we have a symmetric interest corridor and a symmetric distribution of $\tilde{t}$.

a2. If $\bar{\gamma}=\bar{\gamma}_{l}=\bar{\gamma}_{u}$, we have that $\bar{\gamma}=\overline{\bar{\gamma}}$ :

$$
\begin{aligned}
& \bar{\gamma}_{l}=\frac{i^{R O}-i^{D F}}{2(1-G(0))}=\frac{i^{L F}-i^{D F}}{2}=\overline{\bar{\gamma}} \\
& \Rightarrow \frac{i^{R O}-i^{D F}}{i^{L F}-i^{D F}}=1-G(0) .
\end{aligned}
$$

A symmetric interest corridor implies that the LHS of (50) is equal to 0.5. Equality then requires, that also the RHS must equal 0.5 . This will only be the case if we have a symmetric distribution of $\tilde{t}$. Consequently, a symmetric interest corridor and a symmetric distribution of $\tilde{t}$ imply that $\bar{\gamma}_{l}=\overline{\bar{\gamma}}$. As argued in point a1 in this proof, these symmetries also imply that $\bar{\gamma}=\bar{\gamma}_{l}=\bar{\gamma}_{u}$. Consequently, for the symmetric case, we have $\bar{\gamma}=\bar{\gamma}_{l}=$ $\bar{\gamma}_{u}=\overline{\bar{\gamma}}$.

a3. If $\bar{\gamma}=\bar{\gamma}_{l}<\overline{\bar{\gamma}}$, we will have that $\bar{\gamma}_{l}<\bar{\gamma}_{\boldsymbol{u}}$, i.e. we will have an asymmetric interest corridor or an asymmetric distribution of $\tilde{t}$ :

$$
\begin{aligned}
& \bar{\gamma}_{l}=\frac{i^{R O}-i^{D F}}{2(1-G(0))}<\frac{i^{L F}-i^{R O}}{2 G(0)}=\bar{\gamma}_{u} \\
& \Rightarrow \frac{i^{R O}-i^{D F}}{i^{L F}-i^{R O}}<\frac{1-G(0)}{G(0)} .
\end{aligned}
$$

If there is a symmetric interest corridor, the LHS of (52) will be equal to 1 . Then (52) requires the RHS to be larger than 1 , so that there must be an asymmetric distribution of $\tilde{t}$. If there is a symmetric distribution of $\tilde{t}$, the RHS (52) will equal 1 . In this case, the LHS must be smaller than 1, i.e. there must be an asymmetric interest corridor. Consequently, $\bar{\gamma}=\bar{\gamma}_{l}<\overline{\bar{\gamma}}$ implies that we have either an asymmetric interest corridor or an asymmetric distribution of $\tilde{t}$.

a4. Furthermore, $\bar{\gamma}_{l}<\bar{\gamma}_{u}$ implies that $\bar{\gamma}_{l}<\overline{\bar{\gamma}}$ :

$$
\begin{aligned}
& \bar{\gamma}_{l}=\frac{i^{R O}-i^{D F}}{2(1-G(0))}<\frac{i^{L F}-i^{D F}}{2}=\overline{\bar{\gamma}} \\
& \Rightarrow \frac{i^{R O}-i^{D F}}{i^{L F}-i^{D F}}<1-G(0) .
\end{aligned}
$$

If there is a symmetric interest corridor, the LHS of (54) will equal 0.5, i.e. for $\bar{\gamma}_{l}$ to be smaller than $\overline{\bar{\gamma}}, 1-G(0)$ must be larger than 0.5 . Considering the argument given in point a3 in this proof, we can show that this is the case for $\bar{\gamma}_{l}<\bar{\gamma}_{u}$ : A symmetric interest corridor implies that the LHS of (52) equals 1 , which requires the RHS of (52) to be larger than 1 , which means that $(1-G(0))>0.5$. Consequently, (54) is fulfilled, so that $\bar{\gamma}_{l}<\overline{\bar{\gamma}}$ if $\bar{\gamma}_{l}<\bar{\gamma}_{u}$. If there is a symmetric distribution of $\tilde{t}$, the RHS of (54) will be 0.5 , i.e. for $\bar{\gamma}_{l}$ to be smaller than $\overline{\bar{\gamma}},\left(i^{R O}-i^{D F}\right) /\left(i^{L F}-i^{D F}\right)$ must be smaller than 0.5. Considering the argument given in point a 3 in this proof, we can show that this is 
the case for $\bar{\gamma}_{l}<\bar{\gamma}_{u}$ : A symmetric distribution of $\tilde{t}$ implies that the RHS of (52) equals 1 , which requires

$$
\begin{aligned}
& i^{R O}-i^{D F}<i^{L F}-i^{R O} \\
& \Rightarrow i^{R O}-i^{D F}+i^{R O}-i^{D F}<i^{L F}-i^{R O}+i^{R O}-i^{D F} \\
& \Rightarrow 2\left(i^{R O}-i^{D F}\right)<i^{L F}-i^{D F} \\
& \Rightarrow\left(i^{R O}-i^{D F}\right) /\left(i^{L F}-i^{D F}\right)<0.5
\end{aligned}
$$

so that (54) is also fulfilled for a symmetric distribution of $\tilde{t}$.

a5. Furthermore, $\bar{\gamma}_{l}<\bar{\gamma}_{u}$ implies that $\bar{\gamma}_{\boldsymbol{u}}>\overline{\bar{\gamma}}$ :

$$
\begin{aligned}
& \bar{\gamma}_{u}=\frac{i^{L F}-i^{R O}}{2 G(0)}>\frac{i^{L F}-i^{D F}}{2}=\overline{\bar{\gamma}} \\
& \Rightarrow \frac{i^{L F}-i^{R O}}{i^{L F}-i^{D F}}>G(0) .
\end{aligned}
$$

If there is a symmetric interest corridor, the LHS of (60) will be 0.5. i.e. for $\bar{\gamma}_{u}$ to be larger than $\overline{\bar{\gamma}}, G(0)$ must be smaller than 0.5 . This is the case for $\bar{\gamma}_{l}<\bar{\gamma}_{u}$ (see point a3 in this proof): A symmetric interest corridor implies that the LHS of (52) equals 1, i.e. $(1-G(0))>G(0)$ which means that $G(0)<0.5$. Consequently, (60) is fulfilled, so that $\bar{\gamma}_{u}>\overline{\bar{\gamma}}$ if $\bar{\gamma}_{l}<\bar{\gamma}_{u}$. If there is a symmetric distribution of $\tilde{t}$, the RHS of (60) will be 0.5 , i.e. for $\bar{\gamma}_{u}$ to be smaller than $\overline{\bar{\gamma}},\left(i^{L F}-i^{R O}\right) /\left(i^{L F}-i^{D F}\right)$ must be larger than 0.5. Considering the argument given in point a3 in this proof, we can show that this is the case for $\bar{\gamma}_{l}<\bar{\gamma}_{u}$ : A symmetric distribution of $\tilde{t}$ implies that the RHS of (52) equals 1 , which requires

$$
\begin{aligned}
& i^{R O}-i^{D F}<i^{L F}-i^{R O} \\
& \Rightarrow i^{R O}-i^{D F}+i^{L F}-i^{L F}<i^{L F}-i^{R O} \\
& \Rightarrow i^{L F}-i^{D F}<i^{L F}+i^{L F}-i^{R O}-i^{R O} \\
& \Rightarrow i^{L F}-i^{D F}<2\left(i^{L F}-i^{R O}\right) \\
& \Rightarrow 0.5<\left(i^{L F}-i^{R O}\right) /\left(i^{L F}-i^{D F}\right)
\end{aligned}
$$

so that (60) is also fulfilled for a symmetric distribution.

Having clarified the relation between the different $\gamma$, we are now in a position to prove that $R O^{*}>c L^{*}$ and $i^{I B M *}=\underline{i^{I B M}}$ if $\bar{\gamma}=\bar{\gamma}_{l}<\gamma \leq \overline{\bar{\gamma}}$. As $\gamma \leq \overline{\bar{\gamma}}$, we have an active interbank market (see Proposition 1). Consequently, the FOC for optimal borrowing from the refinancing operations is given by $(40)$.

When borrowing $\boldsymbol{R O}=\boldsymbol{c} \boldsymbol{L}, G(\bar{t})$ equal $G(0)$, and the interbank rate, which fulfills (40), is $\tilde{i}^{I B M}$, see proof of Proposition 1 . However, as revealed by (41), for $\gamma>\bar{\gamma}_{l}, \tilde{i}^{I B M} \notin I$, i.e. for $\gamma>\bar{\gamma}_{l}, R O=c L$ cannot be an equilibrium.

When borrowing $\boldsymbol{R O}>\boldsymbol{c L}, i^{I B M}=\underline{i^{I B M}}=i^{D F}+\gamma$, see Proposition 1 and (17). Inserting $\underline{i^{I B M}}$ into (40), we get that borrowing $R O>c L$ is optimal for those $\gamma$ given by (43). These $\gamma$ are strictly larger than $\bar{\gamma}_{l}$, as (44) reveals. As $\bar{\gamma}_{l}<\overline{\bar{\gamma}}$ (see point a4 in this proof), for $\bar{\gamma}_{l}<\gamma \leq \overline{\bar{\gamma}}, \underline{i^{I B M}} \in I$ (see Proposition 1). Consequently, if $\bar{\gamma}=\bar{\gamma}_{l}<\gamma \leq \overline{\bar{\gamma}}$, $R O^{*}>c L^{*}$ and $i^{I B M *}=\underline{i^{I B M}}$ will be an equilibrium.

When borrowing $\boldsymbol{R O} \overline{\langle\boldsymbol{c L}}, i^{I B M}=\overline{i^{I B M}}=i^{L F}-\gamma$, see Proposition 1 and (16). Inserting $\overline{i^{I B M}}$ into (40), we get that borrowing $R O<c L$ is optimal for those $\gamma$ given by (45). These $\gamma$ are strictly larger than $\bar{\gamma}_{u}$, as (46) reveals. As $\bar{\gamma}_{u}>\overline{\bar{\gamma}}$ (see point a5 in this proof), there is no active interbank market for $\gamma>\bar{\gamma}_{u}$, so that $\overline{i^{I B M}} \notin I$. Hence, $R O<c L$ cannot be an equilibrium if $\bar{\gamma}=\bar{\gamma}_{l}<\gamma \leq \overline{\bar{\gamma}}$.

Consequently, if $\bar{\gamma}=\bar{\gamma}_{l}<\gamma \leq \overline{\bar{\gamma}}, R O^{*}>c L^{*}$ and $i^{I B M *}=\underline{i^{I B M}}$ will be the only equilibrium. 
(b) It has to be shown that if $\bar{\gamma}=\bar{\gamma}_{u}<\gamma \leq \overline{\bar{\gamma}}, R O^{*}<c L^{*}$ and $i^{I B M *}=\overline{i^{I B M}}$. It is helpful to have a closer look at those relations between $\bar{\gamma}, \bar{\gamma}_{l}, \bar{\gamma}_{u}$, and $\overline{\bar{\gamma}}$ differing from those described in the proof of Proposition 3(a) first. Note again that we assume that either the interest corridor or the distribution of $\tilde{t}$ is asymmetric or that both are symmetric.

b1. See a1.

b2. See a2.

b3. If $\bar{\gamma}=\bar{\gamma}_{u}<\overline{\bar{\gamma}}$ we will have that $\bar{\gamma}_{\boldsymbol{u}}<\bar{\gamma}_{l}$, i.e. we will have an asymmetric interest corridor or an asymmetric distribution of $\tilde{t}$ :

$$
\begin{aligned}
& \bar{\gamma}_{l}=\frac{i^{R O}-i^{D F}}{2(1-G(0))}>\frac{i^{L F}-i^{R O}}{2 G(0)}=\bar{\gamma}_{u} \\
& \Rightarrow \frac{i^{R O}-i^{D F}}{i^{L F}-i^{R O}}>\frac{1-G(0)}{G(0)} .
\end{aligned}
$$

If there is a symmetric interest corridor, the LHS of (67) will be equal to 1 . Then (52) requires the RHS to be smaller than 1 , implying that there must be an asymmetric distribution of $\tilde{t}$. However, if there is a symmetric distribution of $\tilde{t}$, the RHS (67) will equal 1 . In this case, the LHS must be larger than 1, i.e. there must be an asymmetric interest corridor. Consequently, $\bar{\gamma}=\bar{\gamma}_{u}<\overline{\bar{\gamma}}$ implies that we have either an asymmetric interest corridor or an asymmetric distribution of $\tilde{t}$.

b4. Furthermore, $\bar{\gamma}_{u}<\bar{\gamma}_{l}$ implies that $\bar{\gamma}_{\boldsymbol{u}}<\overline{\bar{\gamma}}$ :

$$
\begin{aligned}
& \bar{\gamma}_{u}=\frac{i^{L F}-i^{R O}}{2 G(0)}<\frac{i^{L F}-i^{D F}}{2}=\overline{\bar{\gamma}} \\
& \Rightarrow \frac{i^{L F}-i^{R O}}{i^{L F}-i^{D F}}<G(0) .
\end{aligned}
$$

If there is a symmetric interest corridor, the LHS of (69) will be 0.5. i.e. for $\bar{\gamma}_{u}$ to be smaller than $\overline{\bar{\gamma}}, G(0)$ must be larger than 0.5. This is the case for $\bar{\gamma}_{u}<\bar{\gamma}_{l}$ (see point b3 in this proof): A symmetric interest corridor implies that the LHS of (67) equals 1 , which requires the RHS of (67) to be smaller than 1, i.e. $(1-G(0))<G(0)$ which means that $G(0)>0.5$. Consequently, (69) is fulfilled, so that $\bar{\gamma}_{u}<\overline{\bar{\gamma}}$ if $\bar{\gamma}_{u}<\bar{\gamma}_{l}$. Note that (69) is also fulfilled for a symmetric distribution of $\tilde{t}$ and an asymmetric interest corridor. The proof is analogous to the one presented in point a4 in this proof.

b5. Furthermore, $\bar{\gamma}_{u}<\bar{\gamma}_{l}$ implies that $\bar{\gamma}_{l}>\overline{\bar{\gamma}}$ :

$$
\begin{aligned}
& \bar{\gamma}_{l}=\frac{i^{R O}-i^{D F}}{2(1-G(0))}>\frac{i^{L F}-i^{D F}}{2}=\overline{\bar{\gamma}} \\
& \Rightarrow \frac{i^{R O}-i^{D F}}{i^{L F}-i^{D F}}>1-G(0) .
\end{aligned}
$$

If there is a symmetric interest corridor, the LHS of (71) will be 0.5 . i.e. for $\bar{\gamma}_{l}$ to be larger than $\overline{\bar{\gamma}},(1-G(0))$ must be smaller than 0.5 . This is the case for $\bar{\gamma}_{u}<\bar{\gamma}_{l}$ (see point b3 in this proof): A symmetric interest corridor implies that the LHS of (67) equals 1, i.e. $(1-G(0))<G(0)$ which means that $(1-G(0))<0.5$. Consequently, (71) is fulfilled, so that $\bar{\gamma}_{l}>\overline{\bar{\gamma}}$ if $\bar{\gamma}_{u}<\bar{\gamma}_{l}$. Note that (71) is also fulfilled for a symmetric distribution of $\tilde{t}$ and an asymmetric interest corridor. The proof is analogous to the one presented in point a5 in this proof.

Having clarified the relation between the different $\gamma$, we are now in a position to proof that $R O^{*}<c L^{*}$ and $i^{I B M *}=\overline{i^{I B M}}$ if $\bar{\gamma}=\bar{\gamma}_{u}<\gamma \leq \overline{\bar{\gamma}}$. As $\gamma \leq \overline{\bar{\gamma}}$, we have an active interbank market (see Proposition 1). Consequently, the FOC for optimal borrowing from the refinancing operations is given by (40).

When borrowing $\boldsymbol{R O}=\boldsymbol{c L}, G(\bar{t})$ equals $G(0)$, and the interbank rate, which fulfills (40), is $\tilde{i}^{I B M}$, see proof of Proposition 1 . However, as revealed by $(42)$, for $\gamma>\bar{\gamma}_{u}, \tilde{i}^{I B M} \notin I$, i.e. for $\gamma>\bar{\gamma}_{u}, R O=c L$ cannot be an equilibrium. 
When borrowing $\boldsymbol{R O}>\boldsymbol{c} \boldsymbol{L}, i^{I B M}=i^{I B M}=i^{D F}+\gamma$, see Proposition 1 and (17). Inserting $\underline{i^{I B M}}$ into (40), we get that borrowing $R O>c L$ is optimal for those $\gamma$ given by (43). These $\gamma$ are strictly larger than $\bar{\gamma}_{l}$ as (44) reveals. As $\bar{\gamma}_{l}>\overline{\bar{\gamma}}$ (see point b5 in this proof), there is no active interbank market for $\gamma>\bar{\gamma}_{l}$, so that $\underline{i^{I B M}} \notin I$, so that $R O>c L$ cannot be an equilibrium if $\bar{\gamma}=\bar{\gamma}_{u}<\gamma \leq \overline{\bar{\gamma}}$.

When borrowing $\boldsymbol{R O}<\boldsymbol{c L}, i^{I B M}=\overline{i^{I B M}}=i^{L F}-\gamma$, see Proposition 1 and (16). Inserting $\overline{i^{I B M}}$ into (40), we get that borrowing $R O<c L$ is optimal for those $\gamma$ given by (45). These $\gamma$ are strictly larger than $\bar{\gamma}_{u}$ as (46) reveals. As $\bar{\gamma}_{u}<\overline{\bar{\gamma}}$ (see point b4 in this proof), for $\bar{\gamma}_{u}<\gamma \leq \overline{\bar{\gamma}}, \overline{i^{I B M}} \in I$ (see Proposition 1). Consequently, if $\bar{\gamma}=\bar{\gamma}_{u}<\gamma \leq \overline{\bar{\gamma}}$, $R O^{*}<c L^{*}$ and $i^{I B M *}=\overline{i^{I B M}}$ will be an equilibrium.

Consequently, if $\bar{\gamma}=\bar{\gamma}_{u}<\gamma \leq \overline{\bar{\gamma}}, R O^{*}<c L^{*}$ and $i^{I B M *}=\overline{i^{I B M}}$ will be the only equilibrium.

\section{A.6 Proof of Proposition 4}

It has to be shown that if $\gamma>\overline{\bar{\gamma}}$,

$$
\begin{array}{lll}
R O^{*}>c L^{*} & \text { if } & G(0)<\left(i^{L F}-i^{R O}\right) /\left(i^{L F}-i^{D F}\right), \\
R O^{*}=c L^{*} & \text { if } & G(0)=\left(i^{L F}-i^{R O}\right) /\left(i^{L F}-i^{D F}\right), \\
R O^{*}<c L^{*} & \text { if } & G(0)>\left(i^{L F}-i^{R O}\right) /\left(i^{L F}-i^{D F}\right) .
\end{array}
$$

As $\gamma>\overline{\bar{\gamma}}$ implies that we have an inactive interbank market (see Proposition 1), the FOC for optimal borrowing from the refinancing operations given in Lemma 1 becomes

$$
\begin{aligned}
& i^{D F} G(\bar{t})+i^{L F}(1-G(\bar{t}))=i^{R O} \\
& \Rightarrow \frac{i^{L F}-i^{R O}}{i^{L F}-i^{D F}}=G(\bar{t}) .
\end{aligned}
$$

Note that we assume that either the interest corridor or the distribution of $\tilde{t}$ is asymmetric or that both are symmetric. If there is a symmetric interest corridor, $\left(i^{L F}-i^{R O}\right) /\left(i^{L F}-i^{D F}\right)=$ 0.5 and (73) requires that $G(\bar{t})=0.5$. Consequently, considering the definition of $\bar{t}$ given by (7), we get from (73):

$$
\begin{aligned}
& \text { if } G(0)=0.5 \Rightarrow G(\bar{t})=G(0) \Rightarrow \bar{t}=0 \Rightarrow R O^{*}=c L^{*}, \\
& \text { if } G(0)>0.5 \Rightarrow G(\bar{t})<G(0) \Rightarrow \bar{t}<0 \Rightarrow R O^{*}<c L^{*}, \\
& \text { if } G(0)<0.5 \Rightarrow G(\bar{t})>G(0) \Rightarrow \bar{t}>0 \Rightarrow R O^{*}>c L^{*} .
\end{aligned}
$$

If there is a symmetric distribution of $\tilde{t}, G(0)=0.5$. Consequently, considering the definition of $\bar{t}$ given by (7), we get from (73):

$$
\begin{aligned}
& \text { if }\left(i^{L F}-i^{R O}\right) /\left(i^{L F}-i^{D F}\right)=0.5 \Rightarrow G(\bar{t})=0.5=G(0) \Rightarrow \bar{t}=0 \Rightarrow R O^{*}=c L^{*}, \\
& \text { if }\left(i^{L F}-i^{R O}\right) /\left(i^{L F}-i^{D F}\right)>0.5 \Rightarrow G(\bar{t})>0.5=G(0) \Rightarrow \bar{t}>0 \Rightarrow R O^{*}>c L^{*}, \\
& \text { if }\left(i^{L F}-i^{R O}\right) /\left(i^{L F}-i^{D F}\right)<0.5 \Rightarrow G(\bar{t})<0.5=G(0) \Rightarrow \bar{t}<0 \Rightarrow R O^{*}<c L^{*} .
\end{aligned}
$$

Therefore, we get that

$$
\begin{array}{lll}
R O^{*}>c L^{*} & \text { if } & G(0)<\left(i^{L F}-i^{R O}\right) /\left(i^{L F}-i^{D F}\right), \\
R O^{*}=c L^{*} & \text { if } & G(0)=\left(i^{L F}-i^{R O}\right) /\left(i^{L F}-i^{D F}\right), \\
R O^{*}<c L^{*} & \text { if } & G(0)>\left(i^{L F}-i^{R O}\right) /\left(i^{L F}-i^{D F}\right) .
\end{array}
$$


A.7 Proof of Result 1

\section{Equilibrium I}

It has to be shown that

$$
\frac{\partial L^{* I}}{\partial \gamma}=\frac{-2 \phi(0)}{\lambda}<0 .
$$

Considering Lemma 3, Proposition 2 and (23), $L^{* I}$ is implicitly defined by

$$
\begin{aligned}
& H(\cdot)^{I}:=-i^{L}+\lambda L^{* I}+c i^{R O}+\phi(0) 2 \gamma, \\
& \text { where } \quad \phi(0)=(1-c) \chi \int_{0}^{t^{\text {max }}} t_{b} g\left(t_{b}\right) d t_{b}, \\
& \text { so that } \quad \frac{\partial H(\cdot)^{I}}{\partial \gamma}=2 \phi(0) \\
& \text { and } \quad \frac{\partial H(\cdot)^{I}}{\partial L^{* I}}=\lambda .
\end{aligned}
$$

Applying the implicit function theorem yields

$$
\frac{\partial L^{* I}}{\partial \gamma}=-\frac{\frac{\partial H(\cdot)^{I}}{\partial \gamma}}{\frac{\partial H(\cdot)^{I}}{\partial L^{* I}}}=-\frac{2 \phi(0)}{\lambda}<0
$$

\section{Equilibrium II}

It has to be shown that

$$
\frac{\partial L^{* I I}}{\partial \gamma}=\left\{\begin{array}{ll}
\frac{2 \phi\left(\bar{t}^{* I I}\right)}{\lambda}+\frac{2(1-c) \chi \bar{t}^{* I I}\left(1-G\left(\bar{t}^{* I I}\right)\right)}{\lambda} & \text { if } \bar{t}^{* I I}>0 \\
-\frac{2 \phi\left(\bar{t}^{* I I}\right)}{\lambda}-\frac{2(1-c) \chi \bar{t}^{* I} \lambda_{G\left(\bar{t}^{* I I}\right)}}{\lambda} & \text { if } \bar{t}^{* I I}<0
\end{array}\right\}<0 .
$$

The proof of (78) is more complex than the proof of $(74)$ as $\bar{t}^{* I I} \neq 0$. Therefore, we divide the proof into four steps.

Step 1: Implicit definition of $L^{* I I}$ by $H(\cdot)^{I I}$, determination of $\partial H(\cdot)^{I I} / \partial L^{* I I}$ and $\boldsymbol{\partial H}(\cdot)^{\boldsymbol{I I}} / \partial \gamma$. Considering Lemma 3, Proposition 3 and (26), $L^{* I I}$ is implicitly defined by

$$
\begin{aligned}
& H(\cdot)^{I I}:=-i^{L}+\lambda L^{* I I}+c i^{R O}+\phi\left(\bar{t}^{* I I}\right) 2 \gamma, \\
& \text { where } \quad \phi\left(\bar{t}^{* I I}\right)=(1-c) \chi \int_{\bar{t}^{* I I}}^{t^{\text {max }}} t_{b} g\left(t_{b}\right) d t_{b}, \\
& \text { and } \quad \bar{t}^{* I I}=\frac{R O^{* I I}\left(\ldots, L^{* I I}, \gamma, \ldots\right)-c L^{* I I}}{(1-c) \chi L^{* I I}} .
\end{aligned}
$$


In Step 4, we will apply the implicit function theorem to obtain $\partial L^{* I I} / \partial \gamma$. Therefore, we determine the first derivatives of $H(\cdot)^{I I}$ with respect to $L^{* I I}$ and $\gamma$ first:

$$
\begin{aligned}
& \frac{\partial H(\cdot)^{I I}}{\partial \gamma}=2 \phi\left(\bar{t}^{* I I}\right)-2 \gamma(1-c) \chi \bar{t}^{* I I} g\left(\bar{t}^{* I I}\right) \frac{\partial \bar{t}^{* I I}}{\partial \gamma}, \\
& \text { with } \quad \frac{\partial \bar{t}^{* I I}}{\partial \gamma}=\frac{\partial \bar{t}^{* I I}}{\partial R O^{* I I}} \frac{\partial R O^{* I I}}{\partial \gamma}=\frac{1}{(1-c) \chi L^{* I I}} \frac{\partial \boldsymbol{R} \boldsymbol{O}^{* I I}}{\partial \gamma} . \\
& \frac{\partial H(\cdot)^{I I}}{\partial L^{* I I}}=\lambda-2 \gamma(1-c) \chi \bar{t}^{* I I} g\left(\bar{t}^{* I I}\right) \frac{\partial \bar{t}^{* I I}}{\partial L^{* I I}}, \\
& \text { with } \frac{\partial \bar{t}^{* I I}}{\partial L^{* I I}}=\frac{\partial \bar{t}^{* I I}}{\partial R O^{* I I}} \frac{\partial R O^{* I I}}{\partial L^{* I I}}=\frac{\left(\frac{\partial \boldsymbol{R} \boldsymbol{O}^{* I I}}{\partial L^{* I I}}-c\right) L^{* I I}-\left(R O^{* I I}-c L^{* I I}\right)}{(1-c) \chi\left(L^{* I I}\right)^{2}} .
\end{aligned}
$$

Step 2: Implicit definition of $R O^{* I I}$ by $Z(\cdot)^{I I}$, determination of $\partial Z(\cdot)^{I I} / \partial \gamma$ $\partial Z(\cdot)^{I I} / \partial L^{* I I}$, and $\partial Z(\cdot)^{I I} / \partial R O^{* I I}$. The first derivatives derived in Step 1 depend on $\partial R O^{* I I} / \partial L^{* I I}$ and $\partial R O^{* I I} / \partial \gamma$, which are determined in this step. Considering Lemma 1 and Proposition $3, R O^{* I I}$ is implicitly defined by

$$
Z(\cdot)^{I I}:= \begin{cases}-i^{R O}+i^{D F}+2 \gamma \int_{\bar{t}^{* I I}}^{t^{\max }} g\left(t_{b}\right) d t_{b} & \text { if } \bar{t}^{* I I}>0, \\ -i^{R O}+i^{L F}-2 \gamma \int_{t^{\min }}^{\bar{t}^{* I I}} g\left(t_{b}\right) d t_{b} & \text { if } \bar{t}^{* I I}<0 .\end{cases}
$$

In order to be able to apply the implicit function theorem to get $\partial R O^{* I I} / \partial L^{* I I}$ and $\partial R O^{* I I} / \partial \gamma$, we determine the first derivatives of $Z(\cdot)^{I I}$ with respect to $R O^{* I I}, L^{* I I}, \gamma$ :

$$
\begin{aligned}
& \frac{\partial Z(\cdot)^{I I}}{\partial \gamma}= \begin{cases}2\left(1-G\left(\bar{t}^{* I I}\right)\right) & \text { if } \bar{t}^{* I I}>0, \\
-2 G\left(\bar{t}^{* I I}\right) & \text { if } \bar{t}^{* I I}<0,\end{cases} \\
& \frac{\partial Z(\cdot)^{I I}}{\partial L^{* I I}}=\frac{2 \gamma R O^{* I I} g\left(\bar{t}^{* I I}\right)}{(1-c) \chi\left(L^{* I I}\right)^{2}} \text { for } \bar{t}^{* I I} \gtrless 0, \\
& \frac{\partial Z(\cdot)^{I I}}{\partial R O^{* I I}}=-\frac{2 \gamma g\left(\bar{t}^{* I I}\right)}{(1-c) \chi L^{* I I}} \text { for } \bar{t}^{* I I} \gtrless 0 .
\end{aligned}
$$

Applying the implicit function theorem yields

$$
\begin{aligned}
& \frac{\partial \boldsymbol{R} \boldsymbol{O}^{* I I}}{\partial \gamma}=-\frac{\frac{\partial Z(\cdot)^{I I}}{\partial \gamma}}{\frac{\partial Z(\cdot)^{I I}}{\partial R O^{* I I}}}= \begin{cases}\frac{2\left(1-G\left(\bar{t}^{* I I}\right)\right)(1-c) \chi L^{* I I}}{2 \gamma g\left(\bar{t}^{* I I}\right)} & \text { if } \bar{t}^{* I I}>0, \\
-\frac{2 G\left(\bar{t}^{* I I}\right)(1-c) \chi L^{* I I}}{2 \gamma g\left(\bar{t}^{* I I}\right)} & \text { if } \bar{t}^{* I I}<0,\end{cases} \\
& \frac{\partial \boldsymbol{R} \boldsymbol{O}^{* I I}}{\partial \boldsymbol{L}^{* I I}}=-\frac{\frac{\partial Z(\cdot)^{I I}}{\partial L^{* I I}}}{\frac{\partial Z(\cdot)^{I I}}{\partial R O^{* I I}}}=\frac{R O^{* I I}}{L^{* I I}} .
\end{aligned}
$$

Step 3: Inserting the results of Step 2 in those of Step 1. Inserting the results for $\partial R O^{* I I} / \partial L^{* I I}$ and $\partial R O^{* I I} / \partial \gamma$ into (83) and (85) gives

$$
\begin{aligned}
& \frac{\partial \bar{t}^{* I I}}{\partial \gamma}= \begin{cases}\frac{1-G\left(\bar{t}^{* I I}\right)}{\gamma g\left(\bar{t}^{* I I}\right)} & \text { if } \bar{t}^{* I I}>0, \\
-\frac{G\left(\bar{t}^{* I I}\right)}{\gamma g\left(\bar{t}^{* I I}\right)} & \text { if } \bar{t}^{* I I}<0,\end{cases} \\
& \frac{\partial \bar{t}^{* I I}}{\partial L^{* I I}}=0,
\end{aligned}
$$

which again means that (82) and (84) (first step) become

$$
\begin{aligned}
& \frac{\partial H(\cdot)^{I I}}{\partial \gamma}= \begin{cases}2 \phi\left(\bar{t}^{* I I}\right)-(1-c) \chi \bar{t}^{* I I} 2\left(1-G\left(\bar{t}^{* I I}\right)\right) & \text { if } \bar{t}^{* I I}>0, \\
2 \phi\left(\bar{t}^{* I I}\right)+(1-c) \chi \bar{t}^{* I I} 2 G\left(\bar{t}^{* I I}\right) & \text { if } \bar{t}^{* I I}<0,\end{cases} \\
& \frac{\partial H(\cdot)^{I I}}{\partial L^{* I I}}=\lambda .
\end{aligned}
$$


Step 4: Applying the implicit function theorem to get $\partial L^{* I I} / \partial \gamma<0$. Applying the implicit function theorem by using (94) and (95) yields

$$
\frac{\partial L^{* I I}}{\partial \gamma}=-\frac{\frac{\partial H(\cdot)^{I I}}{\partial \gamma}}{\frac{\partial H(\cdot)^{I I}}{\partial L^{* I I}}}=\left\{\begin{array}{ll}
-\frac{1}{\lambda}\left[2 \phi\left(\bar{t}^{* I I}\right)-2(1-c) \chi \bar{t}^{* I I}\left(1-G\left(\bar{t}^{* I I}\right)\right)\right] & \text { if } \bar{t}^{* I I}>0 \\
-\frac{1}{\lambda}\left[2 \phi\left(\bar{t}^{* I I}\right)+2(1-c) \chi \bar{t}^{* I I} G\left(\bar{t}^{* I I}\right)\right] & \text { if } \bar{t}^{* I I}<0
\end{array}\right\}<0,
$$

what had to be shown, see (78). However, with respect to the negative sign of $\partial L^{* I I} / \partial \gamma$ we still have to show that the terms in square brackets are positive:

$$
\begin{array}{ll}
2 \phi\left(\bar{t}^{* I I}\right)-2(1-c) \chi \bar{t}^{* I I}\left(1-G\left(\bar{t}^{* I I}\right)\right)>0 & 2 \phi\left(\bar{t}^{* I I}\right)+2(1-c) \chi \bar{t}^{* I I} G\left(\bar{t}^{* I I}\right)>0 \\
\Leftrightarrow \int_{\bar{t}^{* I I}}^{t^{\max }} t_{b} g\left(t_{b}\right) d t_{b}-\bar{t}^{* I I}\left(1-G\left(\bar{t}^{* I I}\right)\right)>0 & \Leftrightarrow \int_{\bar{t}^{* I I}}^{t^{\max }} t_{b} g\left(t_{b}\right) d t_{b}+\bar{t}^{* I I} G\left(\bar{t}^{* I I}\right)>0 \\
\Leftrightarrow \frac{\int_{\bar{t}^{*}}^{t^{\max }} t_{b} g\left(t_{b}\right) d t_{b}}{1-G\left(\bar{t}^{* I I}\right)}>\bar{t}^{* I I} & \Leftrightarrow-\int_{t^{\min }}^{\bar{t}^{* I I}} t_{b} g\left(t_{b}\right) d t_{b}+\bar{t}^{* I I} G\left(\bar{t}^{* I I}\right)>0 \\
\Leftrightarrow E\left[t_{b} \mid t_{b}>\bar{t}^{* I I}\right]>\bar{t}^{* I I} & \Leftrightarrow \bar{t}^{* I I}>\frac{\int_{t^{\text {min }}} t_{b} g\left(t_{b}\right) d t_{b}}{G\left(\bar{t}^{* I I}\right)} \\
& \Leftrightarrow \bar{t}^{* I I}>E\left[t_{b} \mid t_{b}<\bar{t}^{* I I}\right]
\end{array}
$$

As $E\left[t_{b} \mid t_{b}>\bar{t}^{* I I}\right]>\bar{t}^{* I I}$ and $\bar{t}^{* I I}>E\left[t_{b} \mid t_{b}<\bar{t}^{* I I}\right]$, the terms in square brackets are positive, so that $\partial L^{* I I} / \partial \gamma<0$. Note with respect to the second transformation shown in the right hand column that due to $E\left[t_{b}\right]=0$, we have that $\int_{\bar{t}^{* I I}}^{t^{\max }} t_{b} g\left(t_{b}\right) d t_{b}=-\int_{t^{\min }}^{\bar{t}^{* I I}} t_{b} g\left(t_{b}\right) d t_{b}$.

\section{Equilibrium III}

Trivial, inactive interbank market.

\section{A.8 Proof of Result 2}

\section{Equilibrium I}

It has to be shown that

$$
\frac{\partial L^{* I}}{\partial \chi}=-\frac{\phi(0) 2 \gamma}{\lambda \chi}<0 .
$$

Partially differentiating $H(\cdot)^{I}$ given by (75) w.r.t. $\chi$, we get

$$
\frac{\partial H(\cdot)^{I}}{\partial \chi}=(1-c) \int_{0}^{t^{\max }} t_{b} g\left(t_{b}\right) d t_{b} 2 \gamma=\frac{\phi(0) 2 \gamma}{\chi} .
$$

Considering (77) and (97), applying the implicit function theorem yields

$$
\frac{\partial L^{* I}}{\partial \chi}=-\frac{\frac{\partial H(\cdot)^{I}}{\partial \chi}}{\frac{\partial H(\cdot)^{I}}{\partial L^{* I}}}=-\frac{\phi(0) 2 \gamma}{\lambda \chi}<0 .
$$




\section{Equilibrium II}

It has to be shown that

$$
\frac{\partial L^{* I I}}{\partial \chi}=\frac{-\phi\left(\bar{t}^{* I I}\right) 2 \gamma}{\lambda \chi}<0 .
$$

The single steps of this proof are analogous to those of the proof of (78). For a detailed description of the following single steps of this proof we therefore refer the reader to the proof of (78) described in Section A.7 in this appendix.

Step 1: Partially differentiating $H(\cdot)^{I I}$ given by (79) w.r.t. $\chi$, we get

$$
\begin{aligned}
& \frac{\partial H(\cdot)^{I I}}{\partial \chi}=(1-c) 2 \gamma\left(\int_{\bar{t}^{* I I}}^{t^{\max }} t_{b} g\left(t_{b}\right) d t_{b}-\chi \bar{t}^{* I I} g\left(\bar{t}^{* I I}\right) \frac{\partial \bar{t}^{* I I}}{\partial \chi}\right) \\
& \text { where } \frac{\partial \bar{t}^{* I I}}{\partial \chi}=\frac{\partial \bar{t}^{* I I}}{\partial R O^{* I I}} \frac{\partial R O^{* I I}}{\partial \chi}=\frac{\frac{\partial \boldsymbol{R} \boldsymbol{O}^{* I I}}{\partial \chi} \chi-\left(R O^{* I I}-c L^{* I I}\right)}{(1-c) L^{* I I} \chi^{2}} .
\end{aligned}
$$

Step 2: Partially differentiating $Z(\cdot)^{I I}$ given by $(86)$ w.r.t. $\chi$, we get

$$
\frac{\partial Z(\cdot)^{I I}}{\partial \chi}=\frac{g\left(\bar{t}^{* I I}\right) \bar{t}^{* I I} 2 \gamma}{\chi} \text { for } \bar{t}^{* I I} \gtrless 0 .
$$

Considering (89) and (101), applying the implicit function theorem yields

$$
\frac{\partial \boldsymbol{R} \boldsymbol{O}^{* I I}}{\partial \chi}=-\frac{\frac{\partial Z(\cdot)^{I I}}{\partial \chi}}{\frac{\partial Z(\cdot)^{I I}}{\partial R O^{* I I}}}=\bar{t}^{* I I}(1-c) L^{* I I} .
$$

Step 3: Inserting (102) into (100), we get

$$
\frac{\partial \bar{t}^{* I I}}{\partial \chi}=0
$$

and (99) simplifies to

$$
\frac{\partial H(\cdot)^{I I}}{\partial \chi}=\frac{\phi\left(\bar{t}^{* I I}\right) 2 \gamma}{\chi} .
$$

Step 4: Considering (95) and (104), applying the implicit function theorem yields

$$
\frac{\partial L^{* I I}}{\partial \chi}=-\frac{\frac{\partial H(\cdot)^{I I}}{\partial \chi}}{\frac{\partial H(\cdot)^{I I}}{\partial L^{* I I}}}=-\frac{\phi\left(\bar{t}^{* I I}\right) 2 \gamma}{\lambda \chi}<0 .
$$

\section{Equilibrium III}

It has to be shown that

$$
\frac{\partial L^{* I I I}}{\partial \chi}=\frac{-\phi\left(\bar{t}^{* I I I}\right)\left(i^{L F}-i^{D F}\right)}{\lambda \chi}<0 .
$$

The single steps of this proof are analogous to those of the proof of (78). For a detailed description of the following single steps of this proof we therefore refer the reader to the proof of (78) described in Section A.7 in this appendix. 
Step 1: Considering Lemma 3, Proposition 4 and (28), $L^{* I I I}$ is implicitly defined by

$$
\begin{aligned}
& H(\cdot)^{I I I}:=-i^{L}+\lambda L^{* I I I}+c i^{R O}+\phi\left(\bar{t}^{* I I I}\right)\left(i^{L F}-i^{D F}\right), \\
& \text { where } \quad \phi\left(\bar{t}^{* I I I}\right)=(1-c) \chi \int_{\bar{t}^{* I I I}}^{t^{\text {max }}} t_{b} g\left(t_{b}\right) d t_{b} \\
& \text { and } \quad \bar{t}^{* I I I}=\frac{R O^{* I I I}\left(L^{* I I I}, \chi\right)-c L^{* I I I}}{(1-c) \chi L^{* I I I}} .
\end{aligned}
$$

In Step 4, we will apply the implicit function theorem to obtain $\partial L^{* I I I} / \partial \chi$. Therefore, we determine the first derivatives of $H(\cdot)^{I I I}$ with respect to $L^{* I I I}$ and $\chi$ first:

$$
\begin{aligned}
& \frac{\partial H(\cdot)^{I I I}}{\partial \chi}=(1-c)\left(i^{L F}-i^{D F}\right)\left(\int_{\bar{t}^{* I I I}}^{t^{\max }} t_{b} g\left(t_{b}\right) d t_{b}-\chi \bar{t}^{* I I} g\left(\bar{t}^{* I I I}\right) \frac{\partial \bar{t}^{* I I I}}{\partial \chi}\right) \\
& \text { with } \frac{\partial \bar{t}^{* I I I}}{\partial \chi}=\frac{\partial \bar{t}^{* I I I}}{\partial R O^{* I I I}} \frac{\partial R O^{* I I I}}{\partial \chi}=\frac{\frac{\partial \boldsymbol{R} \boldsymbol{O}^{* I I I}}{\partial \chi} \chi-\left(R O^{* I I I}-c L^{* I I I}\right)}{(1-c) L^{* I I I} \chi^{2}} . \\
& \frac{\partial H(\cdot)^{I I I}}{\partial L^{* I I I}}=\lambda-\left(i^{L F}-i^{D F}\right)(1-c) \chi \bar{t}^{* I I I} g\left(\bar{t}^{* I I I}\right) \frac{\partial \bar{t}^{* I I I}}{\partial L^{* I I I}}, \\
& \text { with } \quad \frac{\partial \bar{t}^{* I I I}}{\partial L^{* I I I}}=\frac{\partial \bar{t}^{* I I I}}{\partial R O^{* I I I}} \frac{\partial R O^{* I I I}}{\partial L^{* I I I}}=\frac{\left(\frac{\partial \boldsymbol{R O} \boldsymbol{O}^{* I I I}}{\partial \boldsymbol{L}^{* I I I}}-c\right) L^{* I I I}-\left(R O^{* I I I}-c L^{* I I I}\right)}{(1-c) \chi\left(L^{* I I I}\right)^{2}} .
\end{aligned}
$$

Step 2: Considering Lemma 1 and Proposition 4, $R O^{* I I I}$ is implicitly defined by

$$
Z(\cdot)^{I I I}:=-i^{R O}+i^{D F} \int_{t^{\min }}^{\bar{t}^{* I I I}} g\left(t_{b}\right) d t_{b}+i^{L F} \int_{\bar{t}^{* I I I}}^{t^{\max }} g\left(t_{b}\right) d t_{b} .
$$

Partially differentiating $Z(\cdot)^{I I I}$ w.r.t. $\chi, L^{* I I I}$, and $R O^{* I I I}$ we get

$$
\begin{aligned}
\frac{\partial Z(\cdot)^{I I I}}{\partial \chi} & =\frac{\left(i^{L F}-i^{D F}\right) g\left(\bar{t}^{* I I}\right) \bar{t}^{* I I I}}{\chi}, \\
\frac{\partial Z(\cdot)^{I I I}}{\partial L^{* I I I}} & =\frac{\left(i^{L F}-i^{D F}\right) R O^{* I I I} g\left(\bar{t}^{* I I I}\right)}{(1-c) \chi\left(L^{* I I}\right)^{2}}, \\
\frac{\partial Z(\cdot)^{I I I}}{\partial R O^{* I I I}} & =-\frac{\left(i^{L F}-i^{D F}\right) g\left(\bar{t}^{* I I I}\right)}{(1-c) \chi L^{* I I I}} .
\end{aligned}
$$

Applying the implicit function theorem yields

$$
\begin{aligned}
& \frac{\partial \boldsymbol{R} \boldsymbol{O}^{* I I I}}{\partial \chi}=\bar{t}^{* I I I}(1-c) L^{* I I I} \\
& \frac{\partial \boldsymbol{R} \boldsymbol{O}^{* I I I}}{\partial L^{* I I I}}=-\frac{\frac{\partial Z(\cdot)^{I I I}}{\partial L^{* I I I}}}{\frac{\partial Z(\cdot)^{I I I}}{\partial R O^{* I I I}}}=\frac{R O^{* I I I}}{L^{* I I I}} .
\end{aligned}
$$

Step 3: Inserting (117) and (118) into (110) and (112) yields

$$
\begin{gathered}
\frac{\partial \bar{t}^{* I I I}}{\partial \chi}=0, \\
\frac{\partial \bar{t}^{* I I I}}{\partial L^{* I I I}}=0,
\end{gathered}
$$


and (109) and (111) simplify to

$$
\begin{aligned}
& \frac{\partial H(\cdot)^{I I I}}{\partial \chi}=\frac{\phi\left(\bar{t}^{* I I I}\right)\left(i^{L F}-i^{D F}\right)}{\chi}, \\
& \frac{\partial H(\cdot)^{I I I}}{\partial L^{* I I I}}=\lambda .
\end{aligned}
$$

Step 4: Considering (121) and (122), applying the implicit function theorem yields

$$
\frac{\partial L^{* I I I}}{\partial \chi}=-\frac{\frac{\partial H(\cdot)^{I I I}}{\partial \chi}}{\frac{\partial H(\cdot)^{I I I}}{\partial L^{* I I I}}}=-\frac{\phi\left(\bar{t}^{* I I I}\right)\left(i^{L F}-i^{D F}\right)}{\chi \lambda}<0 .
$$

\section{A.9 Proof of Result 3}

\section{Equilibrium I}

It has to be shown that if $\frac{d i^{D F}}{d i^{R O}}=\frac{d i^{L F}}{d i^{R O}}=1$,

$$
\frac{\partial L^{* I}}{\partial i^{R O}}=-\frac{c}{\lambda}<0
$$

Partially differentiating $H(\cdot)^{I}$ given by (75) w.r.t. $i^{R O}$, we get

$$
\frac{\partial H(\cdot)^{I}}{\partial i^{R O}}=c
$$

Considering (77) and (124), applying the implicit function theorem yields

$$
\frac{\partial L^{* I}}{\partial i^{R O}}=-\frac{\frac{\partial H(\cdot)^{I}}{\partial i^{R O}}}{\frac{\partial H(\cdot)^{I}}{\partial L^{* I}}}=-\frac{c}{\lambda}<0
$$

\section{Equilibrium II}

It has to be shown that if $\frac{d i^{D F}}{d i^{R O}}=\frac{d i^{L F}}{d i^{R O}}=1$,

$$
\frac{\partial L^{* I I}}{\partial i^{R O}}=-\frac{c}{\lambda}<0
$$

The single steps of this proof are analogous to those of the proof of (78). For a detailed description of the following single steps of this proof we therefore refer the reader to the proof of (78) described in Section A.7 in this appendix.

Step 1: Partially differentiating $H(\cdot)^{I I}$ given by $(79)$ w.r.t. $i^{R O}$, we get

$$
\begin{aligned}
& \frac{\partial H(\cdot)^{I I}}{\partial i^{R O}}=c-2 \gamma(1-c) \chi \bar{t}^{* I I} g\left(\bar{t}^{* I I}\right) \frac{\partial \bar{t}^{* I I}}{\partial i^{R O}} \\
& \text { where } \frac{\partial \bar{t}^{* I I}}{\partial i^{R O}}=\frac{\partial \bar{t}^{* I I}}{\partial R O^{* I I}} \frac{\partial R O^{* I I}}{\partial i^{R O}}=\frac{1}{(1-c) \chi L^{* I I}} \frac{\partial \boldsymbol{R O ^ { * I I }}}{\partial \boldsymbol{i}^{R O}} .
\end{aligned}
$$


Step 2: Partially differentiating $Z(\cdot)^{I I}$ given by (86) w.r.t. $i^{R O}$, considering that $\frac{d i^{D F}}{d i^{R O}}=$ $\frac{d i^{L F}}{d i^{R O}}=1$, we get

$$
\frac{\partial Z(\cdot)^{I I}}{\partial i^{R O}}=0 \text { for } \bar{t}^{* I I} \gtrless 0 .
$$

Considering (89) and (128), applying the implicit function theorem yields

$$
\frac{\partial \boldsymbol{R} \boldsymbol{O}^{* I I}}{\partial \boldsymbol{i}^{\boldsymbol{R O}}}=-\frac{\frac{\partial Z(\cdot)^{I I}}{\partial i^{R O}}}{\frac{\partial Z(\cdot)^{I I}}{\partial R O^{* I I}}}=0 .
$$

Step 3: Inserting (129) into (127), we get

$$
\frac{\partial \bar{t}^{* I I}}{\partial i^{R O}}=0
$$

and (126) simplifies to

$$
\frac{\partial H(\cdot)^{I I}}{\partial i^{R O}}=c .
$$

Step 4: Considering (95) and (131), applying the implicit function theorem yields

$$
\frac{\partial L^{* I I}}{\partial i^{R O}}=-\frac{\frac{\partial H(\cdot)^{I I}}{\partial i^{R O}}}{\frac{\partial H(\cdot)^{I I}}{\partial L^{* I I}}}=-\frac{c}{\lambda}<0 .
$$

\section{Equilibrium III}

It has to be shown that if $\frac{d i^{D F}}{d i^{R O}}=\frac{d i^{L F}}{d i^{R O}}=1$,

$$
\frac{\partial L^{* I I I}}{\partial i^{R O}}=-\frac{c}{\lambda}<0 .
$$

The single steps of this proof are analogous to those of the proof of (78). For a detailed description of the following single steps of this proof we therefore refer the reader to the proof of (78) described in Section A.7 in this appendix.

Step 1: Due to $\frac{d i^{D F}}{d i^{R O}}=\frac{d i^{L F}}{d i^{R O}}=1$ it follows from (106) that

$$
\begin{aligned}
& \frac{\partial H(\cdot)^{I I I}}{\partial i^{R O}}=c-\left(i^{L F}-i^{D F}\right)(1-c) \chi \bar{t}^{* I I I} g\left(\bar{t}^{* I I I}\right) \frac{\partial \bar{t}^{* I I I}}{\partial i^{R O}} \\
& \text { where } \frac{\partial \bar{t}^{* I I I} *}{\partial i^{R O}}=\frac{\partial \bar{t}^{* I I I}}{\partial R O^{* I I I}} \frac{\partial R O^{* I I I}}{\partial i^{R O}}=\frac{1}{(1-c) \chi L^{* I I I}} \frac{\boldsymbol{\partial R \boldsymbol { O } ^ { * I I I }}}{\partial \boldsymbol{i}^{R O}}
\end{aligned}
$$

Step 2: Due to $\frac{d i^{D F}}{d i^{R O}}=\frac{d i^{L F}}{d i^{R O}}=1$ it follows from (113) that

$$
\frac{\partial Z(\cdot)^{I I I}}{\partial i^{R O}}=0
$$

Considering (135), applying the implicit function theorem thus yields

$$
\frac{\partial R \boldsymbol{O}^{* I I I}}{\partial i^{R O}}=-\frac{\frac{\partial Z(\cdot)^{I I I}}{\partial i^{R O}}}{\frac{\partial Z(\cdot)^{I I I}}{\partial R O^{* I I I}}}=0 .
$$


Step 3: Inserting (136) into (134), we get

$$
\frac{\partial \bar{t}^{* I I I}}{\partial i^{R O}}=0
$$

and (133) simplifies to

$$
\frac{\partial H(\cdot)^{I I I}}{\partial i^{R O}}=c .
$$

Step 4: Considering (122) and (138), applying the implicit function theorem yields

$$
\frac{\partial L^{* I I I}}{\partial i^{R O}}=-\frac{\frac{\partial H(\cdot)^{I I I}}{\partial i^{R O}}}{\frac{\partial H(\cdot)^{I I I}}{\partial L^{* I I I}}}=-\frac{c}{\lambda}<0 .
$$

\section{A.10 Proof of Result 4}

\section{Equilibrium I}

It has to be shown that if $\frac{d i^{D F}}{d i^{L F}}=-1$,

$$
\frac{\partial L^{* I}}{\partial i^{L F}}=0
$$

Partially differentiating $H(\cdot)^{I}$ given by $(75)$ w.r.t. $i^{L F}$ considering that $\frac{d i^{D F}}{d i^{L F}}=-1$ we get

$$
\frac{\partial H(\cdot)^{I}}{\partial i^{L F}}=0 .
$$

Considering (77), applying the implicit function theorem thus yields

$$
\frac{\partial L^{* I}}{\partial i^{L F}}=-\frac{\frac{\partial H(\cdot)^{I}}{\partial i^{L F}}}{\frac{\partial H(\cdot)^{I}}{\partial L^{* I}}}=0 .
$$

\section{Equilibrium II}

It has to be shown that if $\frac{d i^{D F}}{d i^{L F}}=-1$,

$$
\frac{\partial L^{* I I}}{\partial i^{L F}}=-\frac{(1-c) \chi\left|\bar{t}^{* I I}\right|}{\lambda}<0 .
$$

The single steps of this proof are analogous to those of the proof of (78). For a detailed description of the following single steps of this proof we therefore refer the reader to the proof of (78) described in Section A.7 in this appendix.

Step 1: Due to $\frac{d i^{D F}}{d i^{L F}}=-1$ it follows from (79) that

$$
\begin{aligned}
& \frac{\partial H(\cdot)^{I I}}{\partial i^{L F}}=-2 \gamma(1-c) \chi \bar{t}^{* I I} g\left(\bar{t}^{* I I}\right) \frac{\partial \bar{t}^{* I I}}{\partial i^{L F}} \\
& \text { where } \frac{\partial \bar{t}^{* I I}}{\partial i^{L F}}=\frac{\partial \bar{t}^{* I I}}{\partial R O^{* I I}} \frac{\partial R O^{* I I}}{\partial i^{L F}}=\frac{1}{(1-c) \chi L^{* I I}} \frac{\boldsymbol{\partial R \boldsymbol { O } ^ { * \boldsymbol { I } \boldsymbol { I } }}}{\boldsymbol{\partial \boldsymbol { i } ^ { \boldsymbol { L } \boldsymbol { F } }}} .
\end{aligned}
$$


Step 2: Due to $\frac{d i^{D F}}{d i^{L F}}=-1$ it follows from (86) that

$$
\frac{\partial Z(\cdot)^{I I}}{\partial i^{L F}}=\left\{\begin{array}{lll}
-1 & \text { if } & \bar{t}^{* I I}>0 \\
1 & \text { if } & \bar{t}^{* I I}<0
\end{array}\right.
$$

Considering (89) and (145), applying the implicit function theorem yields

$$
\frac{\partial \boldsymbol{R} \boldsymbol{O}^{* I I}}{\partial \boldsymbol{i}^{\boldsymbol{L} \boldsymbol{F}}}=-\frac{\frac{\partial Z(\cdot)^{I I}}{\partial i^{L F}}}{\frac{\partial Z(\cdot)^{I I}}{\partial R O^{* I I}}}= \begin{cases}-\frac{(1-c) \chi L^{* I I}}{2 \gamma g\left(\bar{t}^{* I I}\right)} & \text { if } \bar{t}^{* I I}>0, \\ \frac{(1-c) \chi L^{* I I}}{2 \gamma g\left(\bar{t}^{* I I}\right)} & \text { if } \bar{t}^{* I I}<0 .\end{cases}
$$

Step 3: Inserting (146) into (144), we get

$$
\frac{\partial \bar{t}^{* I I}}{\partial i^{L F}}= \begin{cases}-\frac{1}{2 \gamma g\left(\bar{t}^{* I I}\right)} & \text { if } \bar{t}^{* I I}>0 \\ \frac{1}{2 \gamma g\left(\bar{t}^{* I I}\right)} & \text { if } \bar{t}^{* I I}<0\end{cases}
$$

and (143) simplifies to

$$
\frac{\partial H(\cdot)^{I I}}{\partial i^{L F}}= \begin{cases}(1-c) \chi \bar{t}^{* I I} & \text { if } \bar{t}^{* I I}>0 \\ -(1-c) \chi \bar{t}^{* I I} & \text { if } \bar{t}^{* I I}<0 .\end{cases}
$$

Step 4: Considering (95) and (148), applying the implicit function theorem thus yields

$$
\begin{aligned}
\frac{\partial L^{* I I}}{\partial i^{L F}} & =-\frac{\frac{\partial H(\cdot)^{I I}}{\partial i^{L F}}}{\frac{\partial H(\cdot)^{I I}}{\partial L^{* I I}}}=\left\{\begin{array}{lll}
-\frac{(1-c) \chi \bar{\chi}^{* I I}}{\lambda} & \text { if } \bar{t}^{* I I}>0 \\
\frac{(1-c) \chi \bar{t}^{* I I}}{\lambda} & \text { if } \bar{t}^{* I I}<0
\end{array}\right\} \\
& =-\frac{(1-c) \chi\left|\bar{t}^{* I I}\right|}{\lambda}<0 \text { for } \bar{t}^{* I I} \gtrless 0 .
\end{aligned}
$$

With respect to the cross derivatives we have

$$
\begin{aligned}
& \frac{\partial^{2} L^{* I I}}{\partial i^{L F} \partial \chi}=\left\{\begin{array}{ll}
-\frac{1-c}{\lambda}\left[\bar{t}^{* I I}+\chi \frac{\partial \bar{t}^{* I I}}{\partial \chi}\right] & \text { if } \bar{t}^{* I I}>0 \\
\frac{1-c}{\lambda}\left[\bar{t}^{* I I}+\chi \frac{\partial \bar{t}^{* I I}}{\partial \chi}\right] & \text { if } \bar{t}^{* I I}<0
\end{array}\right\}=-\frac{(1-c)\left|\bar{t}^{* I I}\right|}{\lambda}<0, \\
& \frac{\partial^{2} L^{* I I}}{\partial i^{L F} \partial \gamma}=\left\{\begin{array}{ll}
-\frac{(1-c) \chi}{\lambda} \frac{\partial \bar{t}^{* I I}}{\partial \gamma}=-\frac{(1-c) \chi\left(1-G\left(\bar{t}^{* I I}\right)\right.}{\lambda \gamma g\left(\bar{t}^{* I I}\right)} & \text { if } \bar{t}^{* I I}>0 \\
\frac{(1-c) \chi}{\lambda} \frac{\partial \bar{t}^{* I I}}{\partial \gamma}=-\frac{(1-c) \chi G\left(\bar{t}^{* I I}\right)}{\lambda \gamma g\left(\bar{t}^{* I I}\right)} & \text { if } \bar{t}^{* I I}<0
\end{array}\right\}<0 .
\end{aligned}
$$

Although $R O^{* I I}$ and $L^{* I I}$ change in $\chi$ (see (102) and (105)) and in $\gamma$ (see (90) and (96)), the change in $L^{* I I}$ does not evoke a change in $\bar{t}^{* I I}$ (see (93)) due to a respective change in $R O^{* I I}$ (see (91)). Consequently, with respect to $\frac{\partial \bar{t}^{* I I}}{\partial \chi} \frac{\partial \bar{t}^{* I I}}{\partial \gamma}$ it will be sufficient to focus on $\frac{\partial \bar{t}^{* I I}}{\partial R O^{* I I}} \frac{\partial R O^{* I I}}{\partial \chi}$ and $\frac{\partial \bar{t}^{* I I}}{\partial R O^{* I T}} \frac{\partial R O^{* I I}}{\partial \gamma}$ given by (100), (103), (83) and (92).

Equilibrium III

It has to be shown that if $\frac{d i^{D F}}{d i^{L F}}=-1$,

$$
\frac{\partial^{2} L^{* I I I}}{\partial i^{L F} \partial \chi}=-\frac{2 \phi\left(\bar{t}^{* I I I}\right)}{\lambda \chi}<0
$$

The single steps of this proof are analogous to those of the proof of (78). For a detailed description of the following single steps of this proof we therefore refer the reader to the 
proof of (78) described in Section A.7 in this appendix.

Step 1: Due to $\frac{d i^{D F}}{d i^{L F}}=-1$ it follows from (106) that

$$
\begin{aligned}
& \frac{\partial H(\cdot)^{I I I}}{\partial i^{L F}}=(1-c) \chi\left[2 \int_{\bar{t}^{* I I I}}^{t^{\max }} t_{b} g\left(t_{b}\right) d t_{b}-\left(i^{L F}-i^{D F}\right) \bar{t}^{* I I} g\left(\bar{t}^{* I I I}\right) \frac{\partial \bar{t}^{* I I I}}{\partial i^{L F}}\right], \\
& \text { where } \frac{\partial \bar{t}^{* I I I}}{\partial i^{L F}}=\frac{\partial \bar{t}^{* I I I}}{\partial R O^{* I I I}} \frac{\partial R O^{* I I I}}{\partial i^{L F}}=\frac{1}{(1-c) \chi L^{* I I I}} \frac{\partial \boldsymbol{R} \boldsymbol{O}^{* \boldsymbol{I I I}}}{\partial \boldsymbol{i}^{\boldsymbol{L} \boldsymbol{F}}} .
\end{aligned}
$$

Step 2: Due to $\frac{d i^{D F}}{d i^{L F}}=-1$ it follows from (113) that

$$
\frac{\partial Z(\cdot)^{I I I}}{\partial i^{L F}}=1-2 G\left(\bar{t}^{* I I I}\right) .
$$

Considering (116) and (156), applying the implicit function theorem yields

$$
\frac{\partial \boldsymbol{R} \boldsymbol{O}^{* \boldsymbol{I I I}}}{\partial \boldsymbol{i}^{\boldsymbol{L F}}}=-\frac{\frac{\partial Z(\cdot)^{I I I}}{\partial i^{L F}}}{\frac{\partial Z(\cdot)^{I I I}}{\partial R O^{* I I I}}}=\frac{\left(1-2 G\left(\bar{t}^{* I I I}\right)\right)(1-c) \chi L^{* I I I}}{\left(i^{L F}-i^{D F}\right) g\left(\bar{t}^{* I I I}\right)}=0 .
$$

Step 3: Inserting (157) into (155) and considering that due to the symmetric interest corridor $G\left(\bar{t}^{* I I I}\right)=1-G\left(\bar{t}^{* I I I}\right)=0.5$ (see $\left.(27)\right)$, we get

$$
\frac{\partial \bar{t}^{* I I I}}{\partial i^{L F}}=0
$$

and (154) simplifies to

$$
\frac{\partial H(\cdot)^{I I I}}{\partial i^{L F}}=2 \phi\left(\bar{t}^{* I I I}\right) .
$$

Step 4: Considering (122) and (159), applying the implicit function theorem thus yields

$$
\frac{\partial L^{* I I I}}{\partial i^{L F}}=-\frac{\frac{\partial H(\cdot)^{I I I}}{\partial i^{L F}}}{\frac{\partial H(\cdot)^{I I I}}{\partial L^{* I I I}}}=-\frac{2 \phi\left(\bar{t}^{* I I I}\right)}{\lambda}<0 .
$$

For the cross derivative we get

$$
\frac{\partial^{2} L^{I I I * I I I}}{\partial i^{L F} \partial \chi}=-\frac{2(1-c)}{\lambda}\left(\int_{\bar{t}^{* I I I}}^{t^{\max }} t_{b} g\left(t_{b}\right) d t_{b}-\chi^{* I I I} g\left(\bar{t}^{* I I I}\right) \frac{\partial \bar{t}^{* I I I}}{\partial \chi}\right)=-\frac{2 \phi\left(\bar{t}^{* I I I}\right)}{\lambda \chi}<0 .
$$

With respect to $\partial \bar{t}^{* I I I} / \partial \chi=0$ (see (119)), see analogously our comments on (151).

\section{A.11 Proof of Result 5}

\section{Equilibrium I}

It has to be shown that

$$
\frac{\partial L^{* I}}{\partial i^{D F}}=0
$$

Partially differentiating $H(\cdot)^{I}$ given by $(75)$ w.r.t. $i^{D F}$ we get

$$
\frac{\partial H(\cdot)^{I}}{\partial i^{D F}}=0
$$


Considering (77) and (163), and applying the implicit function theorem yields

$$
\frac{\partial L^{* I}}{\partial i^{D F}}=-\frac{\frac{\partial H(\cdot)^{I}}{\partial i^{D F}}}{\frac{\partial H(\cdot)^{I}}{\partial L^{* I}}}=0 .
$$

\section{Equilibrium II}

It has to be shown that

$$
\frac{\partial L^{* I I}}{\partial i^{D F}}=\left\{\begin{aligned}
\frac{(1-c) \chi \bar{t}^{* I I}}{\lambda}>0 & \text { if } \bar{t}^{* I I}>0, \\
0 & \text { if } \bar{t}^{* I I}<0 .
\end{aligned}\right.
$$

The single steps of this proof are analogous to those of the proof of (78). For a detailed description of the following single steps of this proof we therefore refer the reader to the proof of (78) described in Section A.7 in this appendix.

Step 1: It follows from (79) that

$$
\begin{aligned}
& \frac{\partial H(\cdot)^{I I}}{\partial i^{D F}}=-2 \gamma(1-c) \chi \bar{t}^{* I I} g\left(\bar{t}^{I I *}\right) \frac{\partial \bar{t}^{* I I}}{\partial i^{D F}}, \\
& \text { where } \frac{\partial \bar{t}^{* I I}}{\partial i^{D F}}=\frac{\partial \bar{t}^{* I I}}{\partial R O^{* I I}} \frac{\partial R O^{* I I}}{\partial i^{D F}}=\frac{1}{(1-c) \chi L^{* I I}} \frac{\partial \boldsymbol{R O ^ { * I I }}}{\partial \boldsymbol{i}^{D \boldsymbol{F}}} .
\end{aligned}
$$

Step 2: It follows from (86) that

$$
\frac{\partial Z(\cdot)^{I I}}{\partial i^{D F}}=\left\{\begin{array}{lll}
1 & \text { if } & \bar{t}^{* I I}>0 \\
0 & \text { if } & \bar{t}^{* I I}<0 .
\end{array}\right.
$$

Considering (89), applying the implicit function theorem thus yields

$$
\frac{\partial \boldsymbol{R} \boldsymbol{O}^{* I I}}{\boldsymbol{\partial \boldsymbol { i } ^ { D \boldsymbol { F } }}}=-\frac{\frac{\partial Z(\cdot)^{I I}}{\partial i^{D F}}}{\frac{\partial Z(\cdot)^{I I}}{\partial R O^{* I I}}}= \begin{cases}\frac{(1-c) \chi L^{* I I}}{2 \gamma g\left(\bar{t}^{* I I}\right)} & \text { if } \bar{t}^{* I I}>0, \\ 0 & \text { if } \bar{t}^{* I I}<0 .\end{cases}
$$

Step 3: Inserting (169) into (167), we get

$$
\frac{\partial \bar{t}^{* I I}}{\partial i^{D F}}= \begin{cases}\frac{1}{2 \gamma g\left(\bar{t}^{* I I}\right)} & \text { if } \bar{t}^{* I I}>0, \\ 0 & \text { if } \bar{t}^{* I I}<0,\end{cases}
$$

and (166) simplifies to

$$
\frac{\partial H(\cdot)^{I I}}{\partial i^{D F}}= \begin{cases}-(1-c) \chi \bar{t}^{* I I} & \text { if } \bar{t}^{* I I}>0, \\ 0 & \text { if } \bar{t}^{* I I}<0 .\end{cases}
$$

Step 4: Considering (95) and (171), applying the implicit function theorem yields

$$
\frac{\partial L^{* I I}}{\partial i^{D F}}=-\frac{\frac{\partial H(\cdot)^{I I}}{\partial i^{D F}}}{\frac{\partial H(\cdot)^{I I}}{\partial L^{* I I}}}= \begin{cases}\frac{(1-c) \chi \bar{t}^{* I I}}{\lambda}>0 & \text { if } \bar{t}^{* I I}>0 \\ 0 & \text { if } \bar{t}^{* I I}<0\end{cases}
$$


which leads to

$$
\begin{aligned}
& \frac{\partial^{2} L^{* I I}}{\partial i^{D F} \partial \chi}= \begin{cases}\frac{1-c}{\lambda}\left[\bar{t}^{* I I}+\chi \frac{\partial \bar{t}^{* I I}}{\partial \chi}\right]=\frac{(1-c) \bar{t}^{* I I}}{\lambda}>0 & \text { if } \bar{t}^{* I I}>0 \\
0 & \text { if } \bar{t}^{* I I}<0 .\end{cases} \\
& \frac{\partial^{2} L^{* I I}}{\partial i^{D F} \partial \gamma}= \begin{cases}\frac{(1-c) \chi}{\lambda} \frac{\partial \bar{t}^{* I I}}{\partial \gamma}=\frac{(1-c) \chi\left(1-G\left(\bar{t}^{* I I}\right)\right)}{\lambda \gamma g\left(\bar{t}^{* I I}\right)}>0 & \text { if } \bar{t}^{* I I}>0, \\
0 & \text { if } \bar{t}^{* I I}<0 .\end{cases}
\end{aligned}
$$

With respect to $\partial \bar{t}^{* I I} / \partial \chi$ and $\partial \bar{t}^{* I I} / \partial \gamma$ see analogously our comments on (151) and (152).

\section{Equilibrium III}

It has to be shown that

$$
\frac{\partial L^{* I I I}}{\partial i^{D F}}=\frac{\phi\left(\bar{t}^{* I I I}\right)}{\lambda}+\frac{(1-c) \chi \bar{t}^{* I I I} G\left(\bar{t}^{* I I I}\right)}{\lambda}>0,
$$

The single steps of this proof are analogous to those of the proof of (78). For a detailed description of the following single steps of this proof we therefore refer the reader to the proof of (78) described in Section A.7 in this appendix.

Step 1: It follows from (106) that

$$
\begin{aligned}
& \frac{\partial H(\cdot)^{I I I}}{\partial i^{D F}}=(1-c) \chi\left[-\int_{\bar{t}^{* I I I}}^{t^{\max }} t_{b} g\left(t_{b}\right) d t_{b}-\left(i^{L F}-i^{D F}\right) \bar{t}^{* I I I} g\left(\bar{t}^{* I I I}\right) \frac{\partial \bar{t}^{* I I I}}{\partial i^{D F}}\right] \\
& \text { where } \frac{\partial \bar{t}^{* I I I}}{\partial i^{D F}}=\frac{\partial \bar{t}^{* I I I}}{\partial R O^{* I I I}} \frac{\partial R O^{* I I I}}{\partial i^{D F}}=\frac{1}{(1-c) \chi L^{* I I I}} \frac{\partial \boldsymbol{R \boldsymbol { O } ^ { * \boldsymbol { I I I } }}}{\partial \boldsymbol{i}^{\boldsymbol{D F}}} .
\end{aligned}
$$

Step 2: It follows from (113) that

$$
\frac{\partial Z(\cdot)^{I I I}}{\partial i^{D F}}=G\left(\bar{t}^{* I I I}\right)
$$

Considering (116), applying the implicit function theorem thus yields

$$
\frac{\partial \boldsymbol{R} \boldsymbol{O}^{* I I I}}{\partial \boldsymbol{i}^{D \boldsymbol{F}}}=-\frac{\frac{\partial Z(\cdot)^{I I I}}{\partial i^{L F}}}{\frac{\partial Z(\cdot)^{I I I}}{\partial R O^{* I I I}}}=\frac{G\left(\bar{t}^{* I I I}(1-c) \chi L^{* I I I}\right.}{\left(i^{L F}-i^{D F}\right) g\left(\bar{t}^{* I I I}\right)} .
$$

Step 3: Inserting (179) into (177), we get

$$
\frac{\partial \bar{t}^{* I I I}}{\partial i^{D F}}=\frac{G\left(\bar{t}^{* I I I}\right)}{\left(i^{L F}-i^{D F}\right) g\left(\bar{t}^{* I I I}\right)}
$$

and (176) simplifies to

$$
\frac{\partial H(\cdot)^{I I I}}{\partial i^{D F}}=-\phi\left(\bar{t}^{* I I I}\right)-(1-c) \chi \bar{t}^{* I I I} G\left(\bar{t}^{* I I I}\right) .
$$

Step 4: Considering (122) and (181), applying the implicit function theorem yields

$$
\begin{aligned}
\frac{\partial L^{* I I I}}{\partial i^{D F}}=-\frac{\frac{\partial H(\cdot)^{I I I}}{\partial i^{D F}}}{\frac{\partial H(\cdot)^{I I I}}{\partial L^{* I I I}}} & =\frac{(1-c) \chi\left[\int_{\bar{t}^{* I I I}}^{t^{\max }} t_{b} g\left(t_{b}\right) d t_{b}+\bar{t}^{* I I I} G\left(\bar{t}^{* I I I}\right)\right]}{\lambda} \\
& =\frac{\phi\left(\bar{t}^{* I I I}\right)+(1-c) \chi \bar{t}^{* I I I} G\left(\bar{t}^{* I I I}\right)}{\lambda}>0,
\end{aligned}
$$


so that

$$
\begin{aligned}
\frac{\partial^{2} L^{* I I I}}{\partial i^{D F} \partial \chi} & =\frac{(1-c)}{\lambda}\left[\left(\int_{\bar{t}^{* I I I}}^{t^{\text {max }}} t_{b} g\left(t_{b}\right) d t_{b}+\bar{t}^{* I I I} G\left(\bar{t}^{* I I I}\right)\right)\right. \\
& +\chi\left(-\bar{t}^{* I I I} g\left(\bar{t}^{* I I I}\right) \frac{\partial \bar{t}^{* I I I}}{\partial \chi}+\frac{\partial \bar{t}^{* I I I}}{\partial \chi} G\left(\left(^{* I I I}\right)-\bar{t}^{* I I I} g\left(\bar{t}^{* I I I}\right) \frac{\partial \bar{t}^{* I I I}}{\partial \chi}\right)\right] \\
& =\frac{\phi\left(\bar{t}^{* I I I}\right)+(1-c) \chi \bar{t}^{* I I I} G\left(\bar{t}^{* I I I}\right)}{\lambda \chi}>0 .
\end{aligned}
$$

With respect to $\partial \bar{t}^{* I I I} / \partial \chi=0$ (see (119)), see analogously our comments on (151). Note that the term $\int_{\bar{t}^{* I I I}}^{t^{\max }} t_{b} g\left(t_{b}\right) d t_{b}+\bar{t}^{* I I I} G\left(\bar{t}^{* I I I}\right)>0$ (see notes to $(96)$ ).

Acknowledgements For helpful comments and suggestions we thank: Aleksander Berentsen, Axel Brüggemann, Falko Fecht, Ilkay Gecgel, Maximilian Horst, Thomas Link, Martin Mandler, Rüdiger Pohl, Franz Seitz, Daniel Stempel, André Sterzel, and an anonymous referee.

\section{Bibliography}

Acharya VV, Merrouche O (2012) Precautionary hoarding of liquidity and interbank markets: Evidence from the sub-prime crisis. Review of Finance 17(1):107-160

Acharya VV, Skeie D (2011) A model of liquidity hoarding and term premia in inter-bank markets. Journal of Monetary Economics 58(5):436-447

Afonso G, Lagos R (2015) Trade dynamics in the market for federal funds. Econometrica 83(1):263-313

Allen F, Carletti E, Gale D (2009) Interbank market liquidity and central bank intervention. Journal of Monetary Economics 56(5):639-652

Alper K, Kara H, Yörükoğlu M (2013) Alternative tools to manage capital flow volatility. Market Volatility and Foreign Exchange Intervention in EMEs: What Has Changed? BIS Papers, No 73 pp 335-352

Alvarez I, Casavecchia F, Luca MD, Duering A, Eser F, Helmus C, Hemous C, Herrala N, Jakovicka J, Russo ML, Pasqualone F, Rubens M, Soares R, Zennaro F (2017) The Use of the Eurosystem's Monetary Policy Instruments and Operational Framework since 2012. European Central Bank Occasional Paper Series No. 188

Andreu Mas-Colell and Michael D Whinston and Jerry R Green (1995) Miroeconomic Theory. Oxford University Press, Oxford (UK)

Ashcraft A, McAndrews J, Skeie D (2011) Precautionary reserves and the interbank market. Journal of Money, Credit, and Banking 43(7):311-348

Ayuso J, Repullo R (2001) Why did the banks overbid? an empirical model of the fixed rate tenders of the european central bank. Journal of International Money and Finance 20(6):857-870

Ayuso J, Repullo R (2003) A model of the open market operations of the european central bank. Economic Journal 113(490):883-902

Baldo L, Hallinger B, Helmus C, Herrala N, Martins D, Mohing F, Petroulakis F, Resinek M, Vergote O, Usciati B, Wang Y (2017) The Distribution of Excess Liquidity in the Euro Area. European Central Bank Occasional Paper Series No. 200

Bank for International Settlements, Committee on the Global Financial System (2015) Regulatory change and monetary policy, CGFS Papers No. 54

Bartolini L, Bertola G, Prati A (2001) Banks' reserve management, transaction costs, and the timing of federal reserve intervention. Journal of Banking and Finance 25(7):12871317

Bartolini L, Bertola G, Prati A (2002) Day-to-day monetary policy and the volatility of the federal funds interest rate. Journal of Money, Credit, and Banking 34(1):137-159

Bech M, Monnet C (2016) A search-based model of the interbank money market and monetary policy implementation. Journal of Economic Theory 164(July):32-67 
Bech ML, Keister T (2013) Liquidity regulation and the implementation of monetary policy, BIS Working Papers No. 432

Berentsen A, Monnet C (2008) Monetary policy in a channel system. Journal of Monetary Economics 55(6):1067-1080

Bernanke BS, Gertler M (1989) Agency costs, net worth, and business fluctuations. American Economic Review 79(1):14-33

Bernanke BS, Gertler M, Gilchrist S (1999) The financial accelerator in a quantitative business cycle framework. In: Taylor JB, Woodford M (eds) Handbook of Macroeconomics, Elsevier, Amsterdam, pp 1341-1393

Bindseil U (2005) Over- and underbidding in central bank open market operations conducted as fixed rate tender. German Economic Review 6(1):95-130

Bindseil U (2014) Monetary Policy Operations and the Financial System. Oxford University Press, Oxford

Bindseil U (2016) Evaluating monetary policy operational frameworks, Paper for presentation at the Jackson Hole Economic Policy Symposium 2016, Federal Reserve Bamk of Kansas City.

Bindseil U, Camba-Mendez G, Hirsch A, Weller B (2006) Excess reserves and the implementation of monetary policy of the ecb. Journal of Policy Modeling 28(5):491-510

Boivin J, Kiley MT, Mishkin FS (2010) How has the monetary transmission mechanism evolved over time? In: Friedman BM, Woodford M (eds) Handbook of Monetary Economics, Elsevier, pp 369-422

Borio C, Disyatat P (2009) Unconventional monetary policy: An appraisal. BIS Working Papers No. 292, Bank for International Settlements

Bräuning F, Fecht F (2017) Relationship Lending in the Interbank Market and the Price of Liquidity. Review of Finance 21(1):33-75

Campbell JY (1987) Money announcements, the demand for bank reserves, and the behavior of the federal funds rate within the statement week. Journal of Money, Credit, and Banking 19(1):56-67

Cassola N, Huetl M (2010) The euro overnight interbank market and ECB's liquidity management policy during tranquil and turbulent times. Paper presented at the ECB Workshop on "Challenges to Monetary Policy Implementation beyond the Financial Market Turbulence", in Frankfurt/Main, Germany on 1 December 2009

Cheun S, von Köppen-Mertes I, Weller B (2009) The collateral frameworks of the Eurosystem, the Federal Reserve System and the Bank of England and the financial market turmoil. European Central Bank Occasional Paper Series No. 107

Clouse JA, Dow JP (1999) Fixed costs and the behaviour of the federal funds rate. Journal of Banking and Finance 23(7):1015-1029

Clouse JA, Dow JP (2002) A computational model of banks' optimal reserve management policy. Journal of Economic Dynamics and Control 26(11):1787-1814

Colliard JE, Foucault T, Hoffmann P (2017) Interbank trading in a segmented OTC market, mimeo

Disyatat P (2011) The bank lending channel revisited. Journal of Money, Credit and Banking 43(4):711-734

Eisenschmidt J, Hirsch A, Linzert T (2009) Bidding behaviour in the ECB's main refinancing operations during the financial crisis, ECB Working Paper No. 1052

Eser F, Amaro MC, Iacobelli S, Rubens M (2012) The Use of the Eurosystem's Monetary Policy Instruments and Operational Framework since 2009. European Central Bank Occasional Paper Series No. 135

European Central Bank (2002) The liquidity management of the ECB. ECB Monthly Bulletin, May 2002 pp 41-54

European Central Bank (2009) The implementation of monetary policy since August 2007. ECB Monthly Bulletin, July 2009 pp 75-89

European Central Bank (2010) The ECB's response to the financial crisis. ECB Monthly Bulletin, October 2010 pp 59-74

European Central Bank (2011) The ECB's non-standard measures - impact and phasing-out. ECB Monthly Bulletin, July 2011 pp 55-69

European Central Bank (2012a) The implementation of monetary policy in the euro area. General documentation on Eurosystem monetary policy instruments and procedures. Eu- 
ropean Central Bank, Frankfurt

European Central Bank (2012b) Monetary policy measures decided by the governing council on 6 September 2012. ECB Monthly Bulletin, September 2012 pp 7-11

European Central Bank (2014) The targeted longer-term refinancing operation of September 2014. ECB Monthly Bulletin, October 2014 pp 21-23

European Central Bank (2015) The governing council's Expanded Asset Purchase Programme. ECB Economic Bulletin, February 2015 pp 15-18

Ewerhart C (2002) A model of the Eurosystem's operational framework for monetary policy implementation, ECB Working Paper No. 197

Freixas X, Jorge J (2008) The role of interbank markets in monetary policy: A model with rationing. Journal of Money, Credit and Banking 40(6):1151-1176

Friedman BM, Kuttner KN (2011) Implementation of monetary policy: How do central banks set interest rates? In: Friedman BM, Woodford M (eds) Handbook of Monetary Economics, Elsevier, pp 1345-1438

Frutos JC, Garcia-de Andoain C, Heider F, Papsdorf P (2016) Stressed interbank markets: Evidence from the European financial and sovereign debt crisis, European Central Bank Working Paper No. 1925

Furfine CH (2000) Interbank payments and the daily federal funds rate. Journal of Monetary Economics 46(2):535-553

Gertler M, Gilchrist S (1993) The role of credit market imperfections in the monetary transmission mechanism: Arguments and evidence. Scandinavian Journal of Economics 95(1):43-64

Hamilton JD (1996) The daily market for federal funds. Journal of Political Economy 104(1):26-56

Hauck A, Neyer U (2014) A model of the Eurosystem's operational framework and the euro overnight interbank market. European Journal of Political Economy 34(June):S65-S82

Heider F, Hoerova M, Holthausen C (2015) Liquidity hoarding and interbank market rates: The role of counterparty risk. Journal of Financial Economics 118(2):336-354

Ho TSY, Saunders A (1985) A micro model of the federal funds market. Journal of Finance 40(3):977-986

Iyer R, Peydró JL, da Rocha-Lopes S, Schoar A (2014) Interbank liquidity crunch and the firm credit crunch: Evidence from the 2007-2009 crisis. Review of Financial Studies $27(1): 347-372$

Jackson C, Noss J (2015) A heterogeneous agent model for assessing the effects of capital regulation on the interbank money market under a corridor system. Bank of England Staff Working Paper No. 548

Kara H (2016) A brief assessment of turkey's macroprudential policy approach: 2011-2015. Central Bank Review 16:85-92

Kashyap AK, Stein JC (1995) The impact of monetary policy on bank balance sheets. Carnegie-Rochester Conference Series on Public Policy 42:151-195

Küçük H, Özlü P, Talasli IA, Ünalmis D, Yüksel C (2016) Interest rate corridor, liquidity mangement, and the overnight spread. Contemprary Economic Policy 34(4):746-761

Lenza M, Pill H, Reichlin L (2010) Monetary policy in exceptional times. Economic Policy 25(62):295-339

Link T, Neyer U (2017) Friction-induced interbank rate volatility under alternative interest corridor systems, Heinrich Heine University Düsseldorf, DICE Discussion Paper No. 275

Nautz D (1998) Banks' demand for reserves when future monetary policy is uncertain. Journal of Monetary Economics 42(1):161-183

Nautz D, Oechssler J (2003) The repo auctions of the European Central Bank and the vanishing quota puzzle. Scandinavian Journal of Economics 105(2):207-220

Nautz D, Oechssler J (2006) Overbidding in fixed rate tenders - an empirical assessment of alternative explanations. European Economic Review 50(3):631-646

Neyer U (2009) Interest on reserves and the flexibility of monetary policy in the euro area. Scandinavian Journal of Economics 111(2):417-438

Neyer U, Wiemers J (2004) The influence of a heterogeneous banking sector on the interbank market rate in the euro area. Swiss Journal of Economics and Statistics 140(3):395-428

Peek J, Rosengreen ES (2012) The role of banks in the transmission of monetary policy. In: Berger AN, Molyneux P, Wilson JO (eds) The Oxford Handbook of Banking, Oxford 
University Press, Oxford, pp 257-277

Pérez-Quirós G, Rodríguez-Mendizábal H (2006) The daily market for funds in Europe: What has changed with the EMU? Journal of Money, Banking, and Credit 38(1):91-110

Poole W (1968) Commercial bank reserve management in a stochastic model: Implications for monetary policy. Journal of Finance 23(5):769-791

Välimäki T (2001) Fixed rate tenders and the overnight money market equilibrium, Bank of Finland Discussion Paper No. 8/2001

Vollmer U, Wiese H (2016) Central bank standing facilities, counterparty risk, and otcinterbank lending. North American Journal of Economics and Finance 36:101-122

Whitesell W (2006) Interest rate corridors and reserves. Journal of Monetary Economics 53(6):1177-1195 